\title{
Distributed Artificial Intelligence for Distributed Corporate Knowledge Management
}

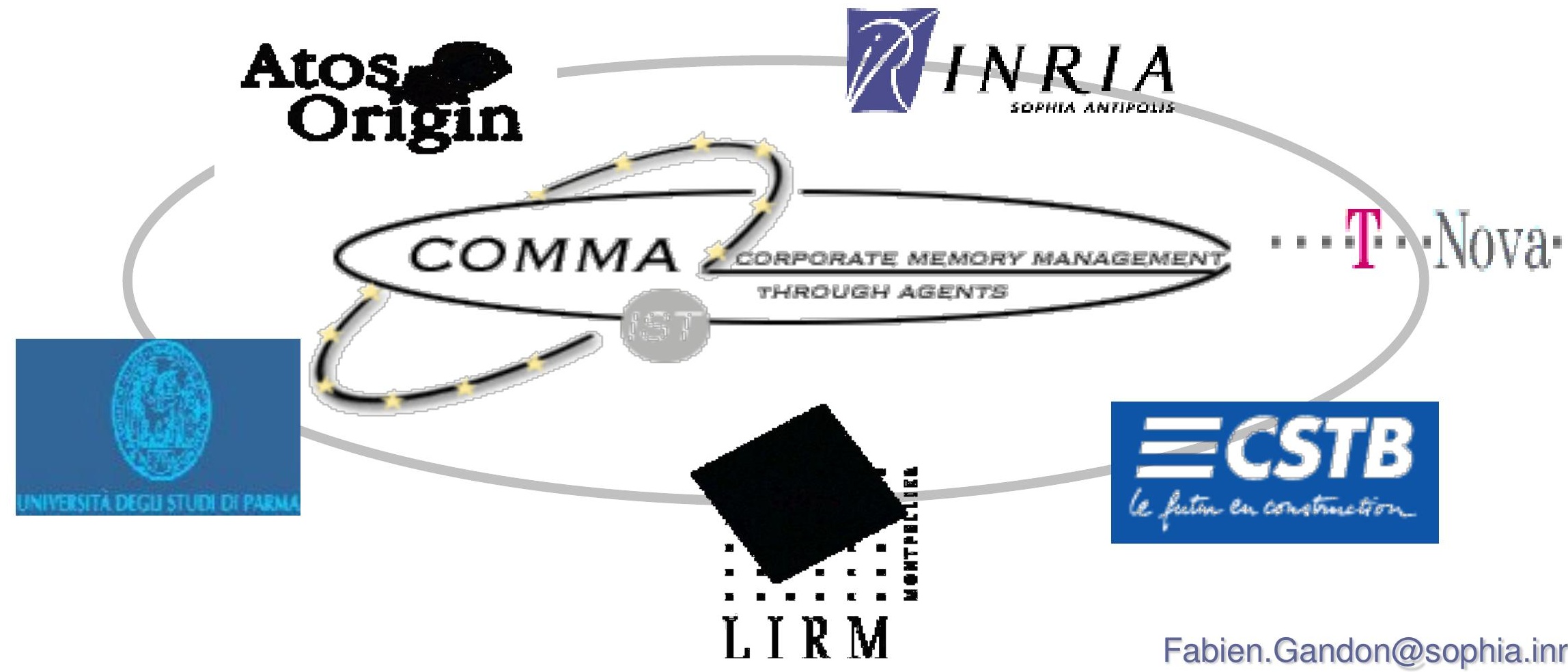

Fabien.Gandon@sophia.inria.fr 
- Dynamically integrating heterogeneous sources of information: OBSERVER [Mena et al., 1996] ; InfoSleuth [Nodine et al., 1999] ; Carnot [Collet et al., 1991] ; InfoMaster [Genesereth et al., 1997] ; SIMS [Arens et al., 1996] ; RETSINA [Decker and Sycara, 1997] ; Manifold [Kirk et al.,1995]

- Digital libraries: SAIRE [Odubiyi et al., 1997] UMDL [Weinstein et al., 1999]

- Knowledge management:

- Collaborative gathering, filtering and profiling: CASMIR [Berney and Ferneley, 1999]; Ricochet [Bothorel and Thomas, 1999]

- Mobile access to memory and domain model for classification: KnowWeb [Dzbor et al., 2000]

- Taxonomy, profiling and push: RICA [Aguirre et al., 2000]

- Ontology and corporate memory:

FRODO [Van Elst and Abecker, 2001] 


\section{- Knowledge and information management:}

- Needs: improve reaction time \& address turnover - Persistent memory: store and/or index knowledge

- Nervous system: capture and diffuse knowledge

- O.M.: an explicit and persistent representation and/or indexing of knowledge in an organization, in order to facilitate its access and reuse by members of the organization, for their individual and collective tasks.

- Current trend: reuse internet and web technologies to build intranets and intrawebs

- Same advantages: standardised technology, browser unique access means, distributed architecture, etc.

- Same drawbacks: human-understandable but only machine readable; problem of retrieval, automation, etc.

- Corporate Memory Management through Agents:

- Assist new employee integration

- Support technology monitoring activities 


\section{- In CoMMA:}

- Corporate memories are heterogeneous and distributed information landscapes

- Stakeholders are an heterogeneous and distributed population

- Exploitation of CM involves heterogeneous and distributed tasks

- Choices:

\begin{tabular}{|c|c|}
\hline Materialization CM & Exploitation CM \\
\hline $\begin{array}{l}\text { XML: Standard, } \\
\text { Structure, Extensible, } \\
\text { Validate, Transform }\end{array}$ & $\begin{array}{l}\text { Multi-Agent System: } \\
\text { Modularity, Distributed, } \\
\text { Collaboration }\end{array}$ \\
\hline $\begin{array}{l}\text { RDF: Annotation, } \\
\text { Schemas }\end{array}$ & $\begin{array}{l}\text { Machine Learning: } \\
\text { Adaptation, Emergence }\end{array}$ \\
\hline
\end{tabular}




\section{- This is not a presentation of...}

- The overall design rationale of CoMMA (see IFIP 2002)

- The top-down organisational function analysis and implementation of the MAS (see AT2AI 2002)

- The ontology design (see ISMICK 2001)

- The machine learning (see PKDD 2001)

- The semantic search engine based on conceptual graphs (see WWW 2002 - RDF workshop)

- Pseudo-semantic distance and distributed query-solving protocols (see AAMAS 2002)

- This is an overview a the CoMMA solution as it was in the last trial:

- The corporate semantic web (distributed knowledge)

- The multi-agent architecture (distributed Al) 


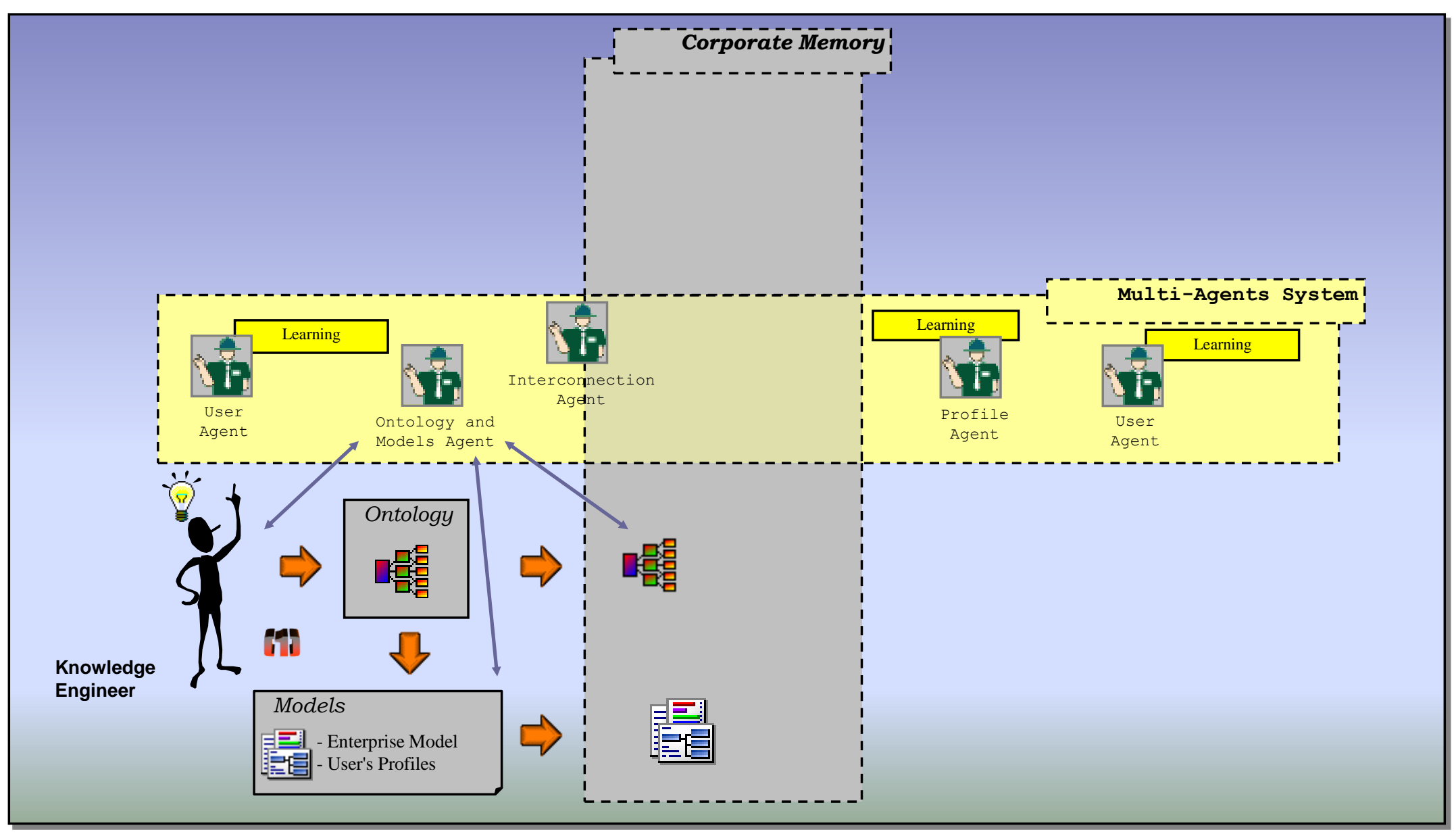

Fabien.Gandon@sophia.inria.fr 


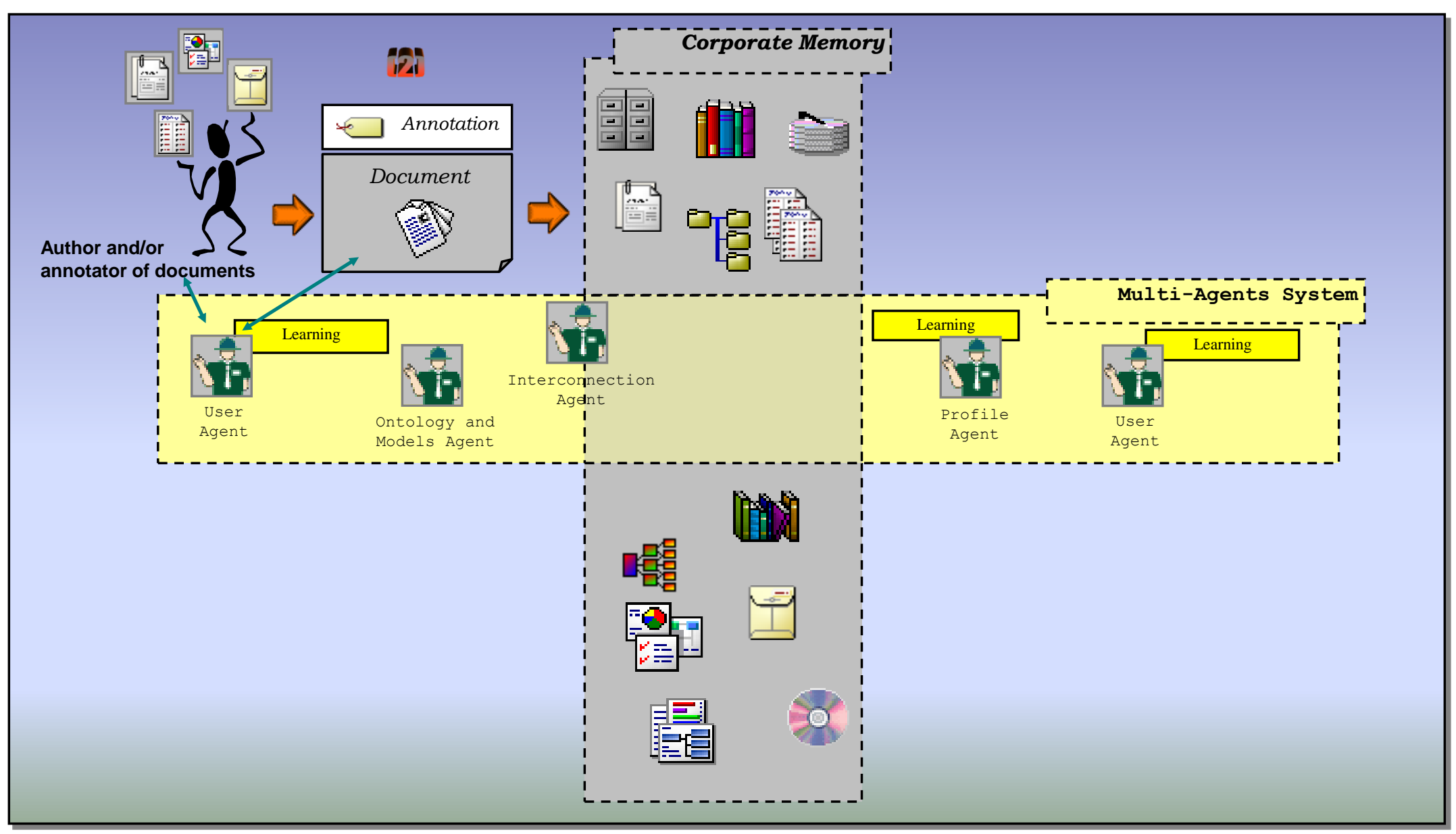

Fabien.Gandon@sophia.inria.fr 


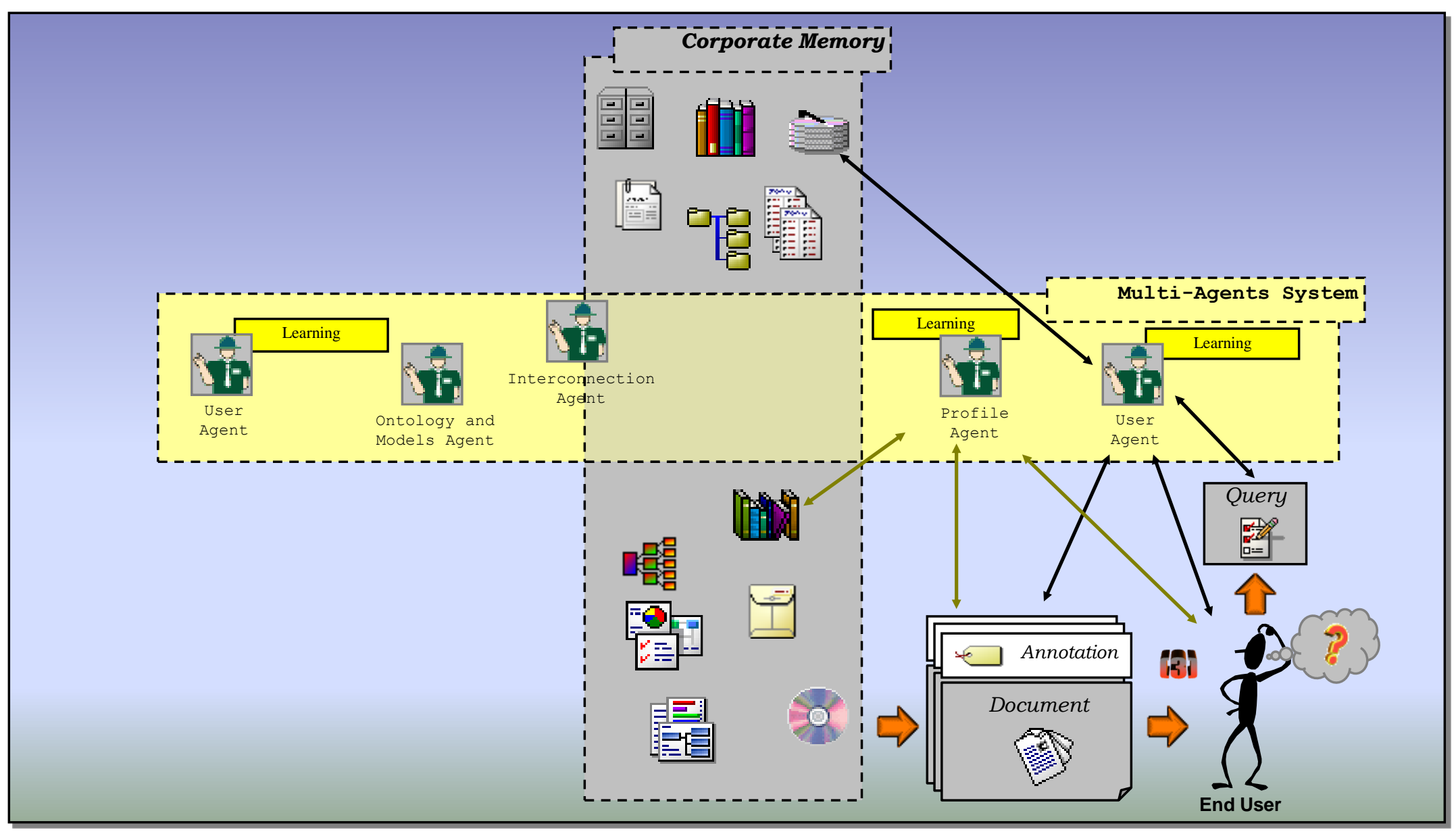

Fabien.Gandon@sophia.inria.fr 


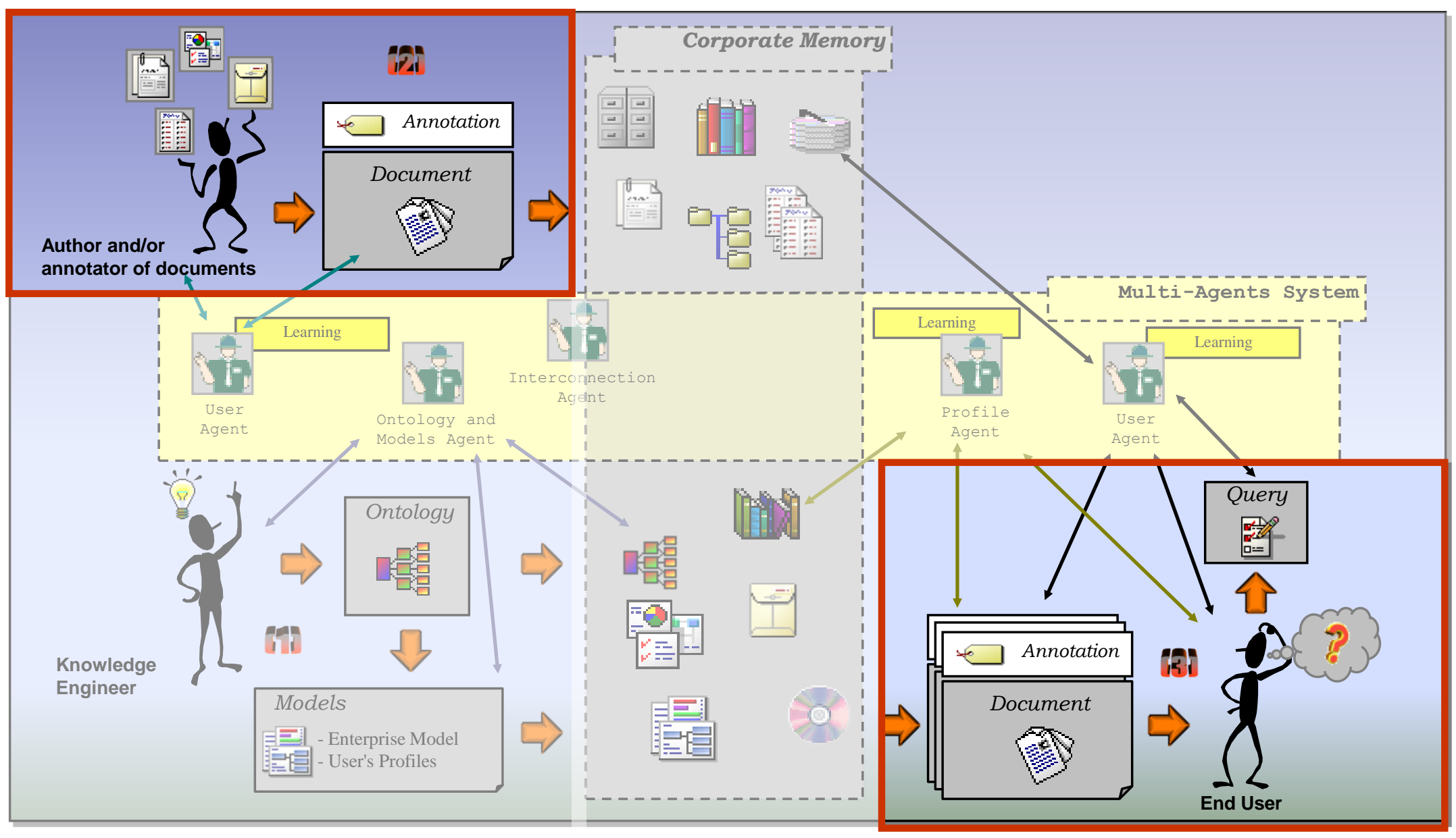

Fabien.Gandon@sophia.inria.fr 


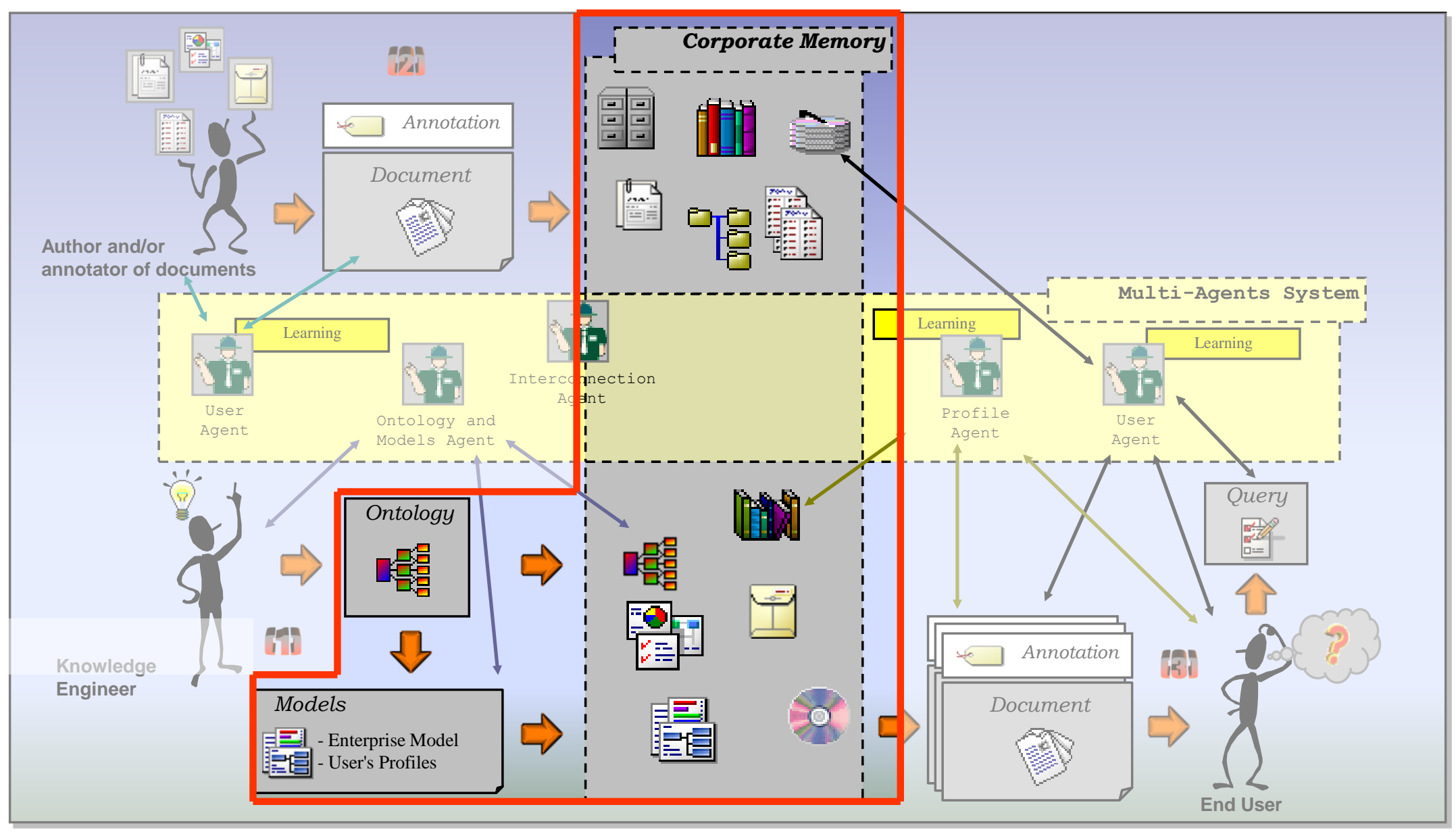

Fabien.Gandon@sophia.inria.fr 
- Ontology: explicit partial account of concepts used in the corporate memory management scenarios and their relations

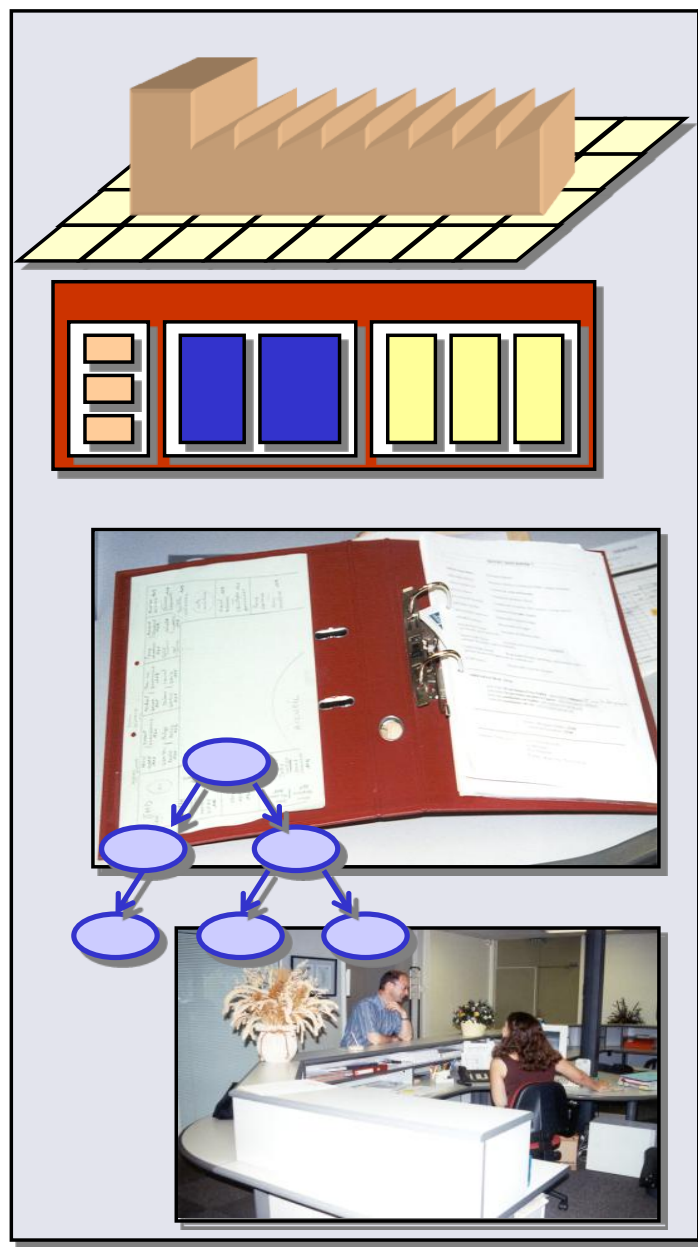

$\square \square$ a - Reality

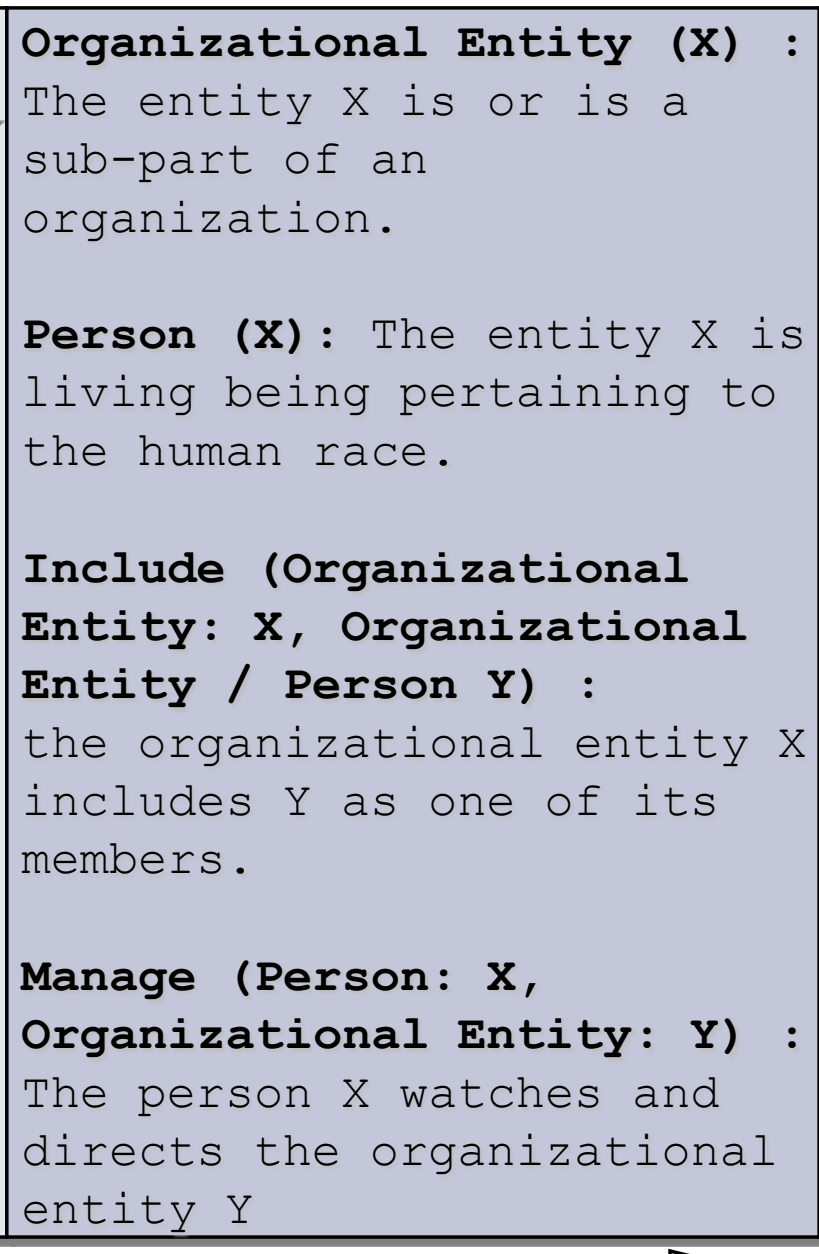

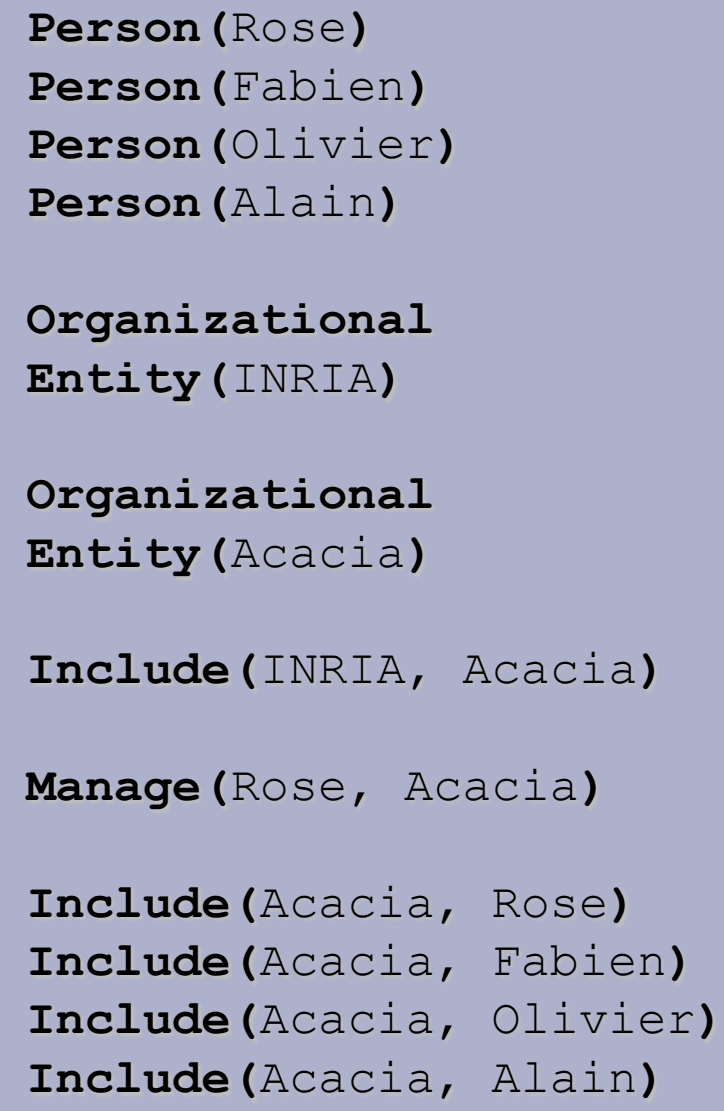

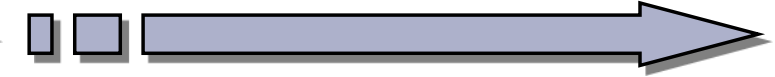

c - Situation \& Annotations 
- XML leitmotiv: Bring structure to the memory to improve search and manipulation of documents using an emerging standard in industry.

- RDF leitmotiv: If the corporate memory becomes an annotated world, software can use the semantics of these annotations an through inferences help the users exploit the corporate memory.

- Corporate semantic web: (annotated info world)

- Ontology in RDFS (O'CoMMA)

- Description the Situation in RDF:

- User Profiles (annotate person)

- Organization model (annotate groups)

- Annotations in RDF describing Documents (manipulation at semantic level)

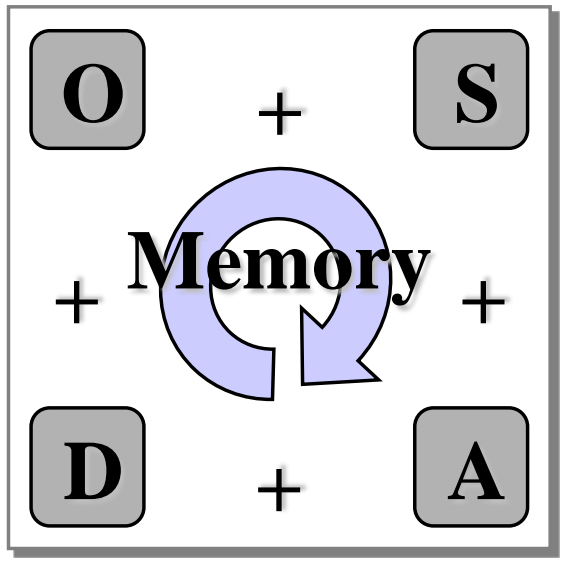

- Annotated persons \& organizational entities

$\rightarrow$ context awareness 


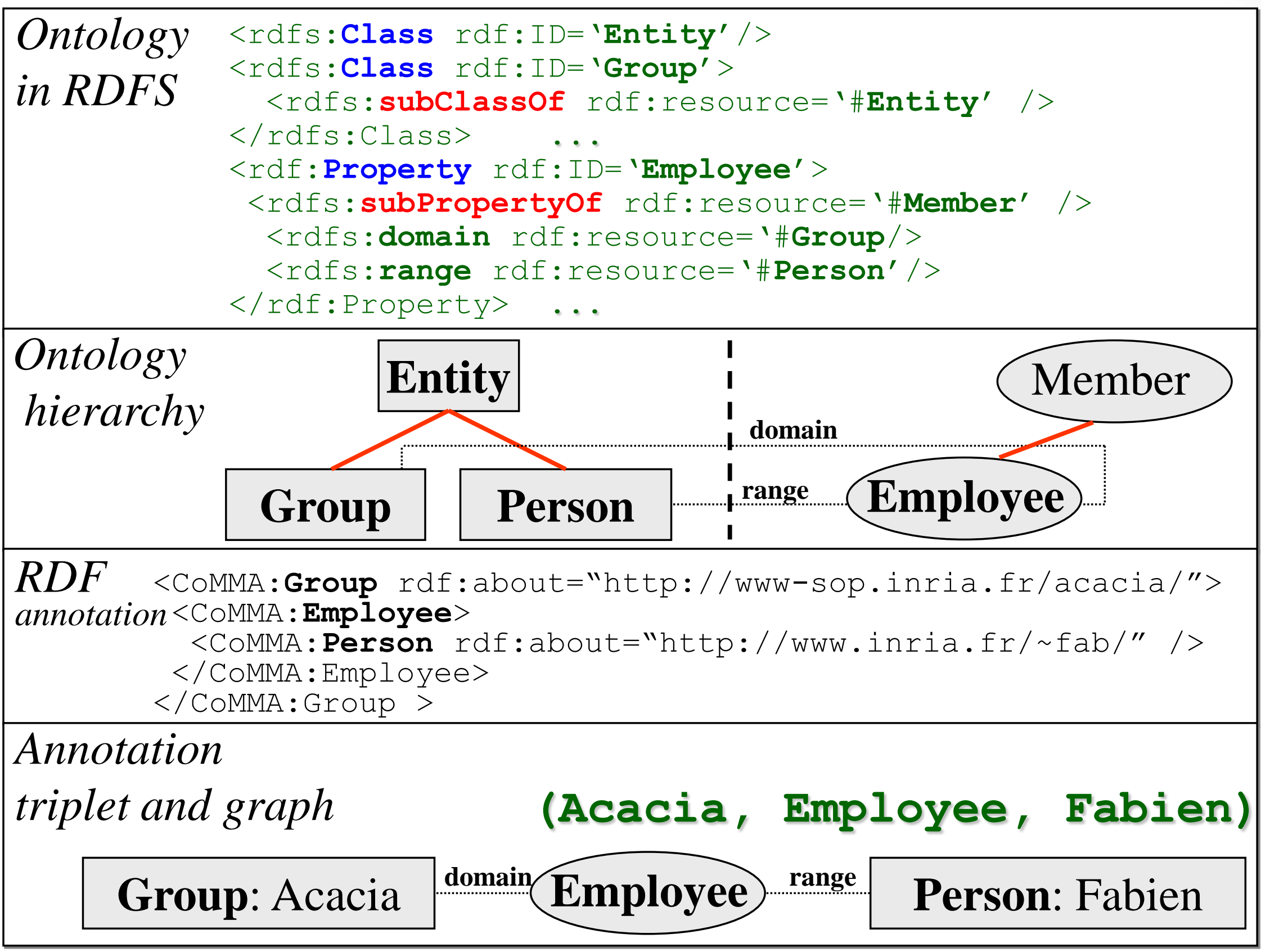




\section{- CORESE: COnceptual REsources Search Er
- RDF(S) for schema, annotations, rules ${ }^{(+)}$, queries ${ }^{(+)}$ \\ - Light weight component \& API}

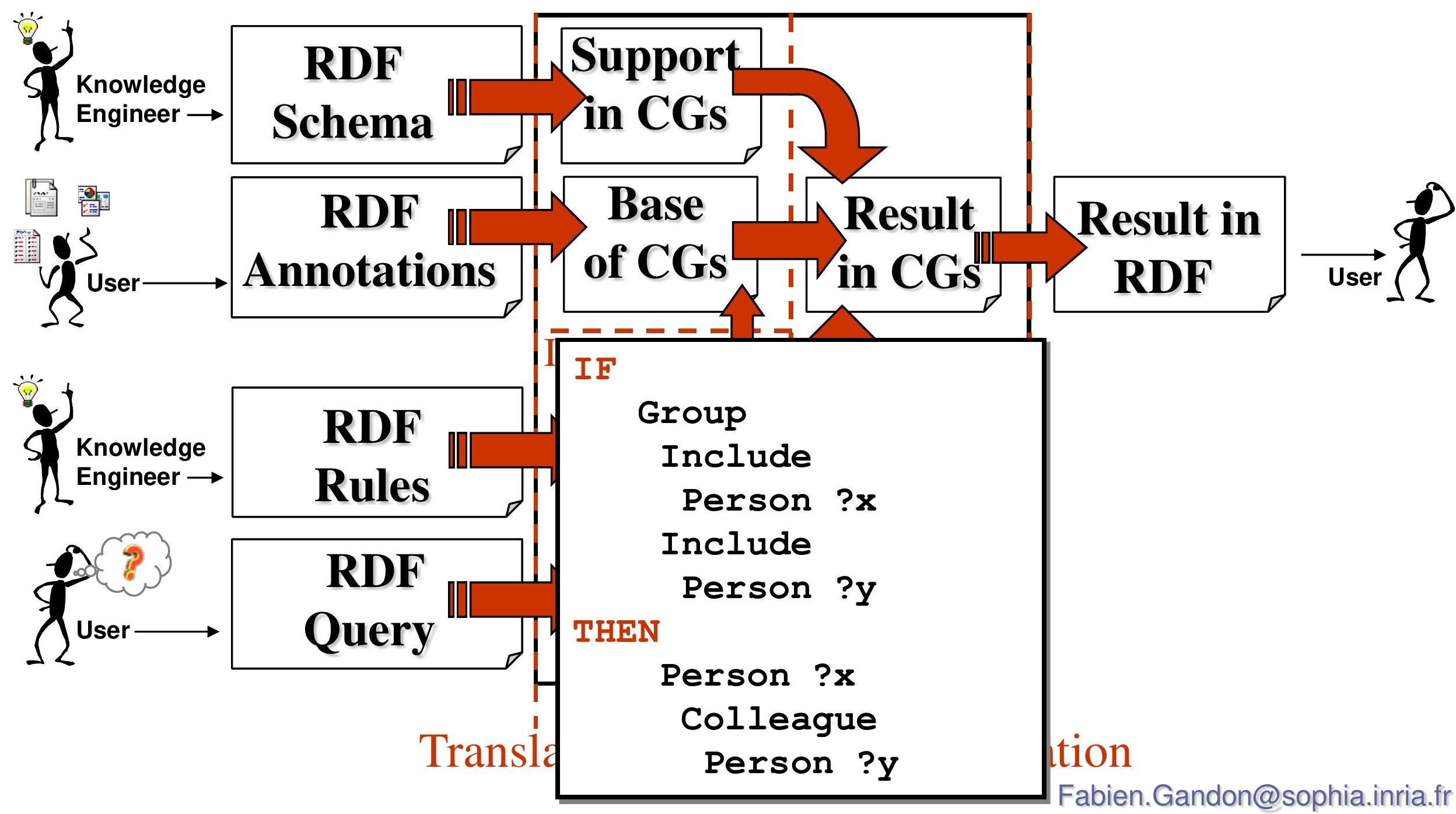




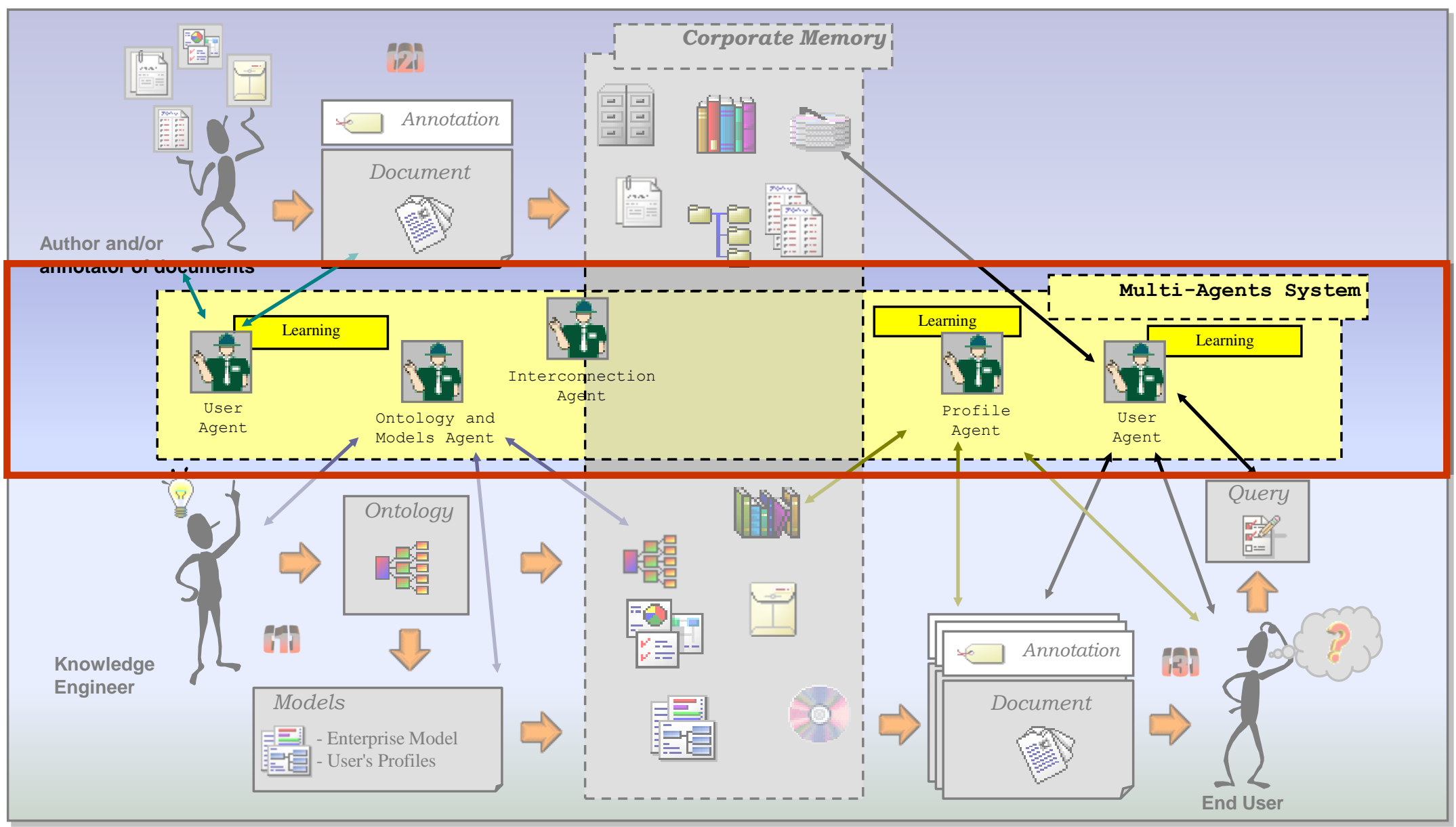

Fabien.Gandon@sophia.inria.fr 
- Leitmotiv: One functional architecture leading to several possible configurations in order to adapt to the broad range of environments that can be found in a company

- Architecture: Agent kinds and their relationships

Fixed at design time

- Configuration: Exact topography of a given MAS Fixed at deployment time

- One architecture $\rightarrow$ Several configurations

Adapt to context

- Agent paradigm adequacy:

- Agent collaboration $\rightarrow$ Global capitalization

- Agent autonomy \& individuality $\rightarrow$ Local adaptation

- CoMMA is an heterogeneous multi-agents information system 


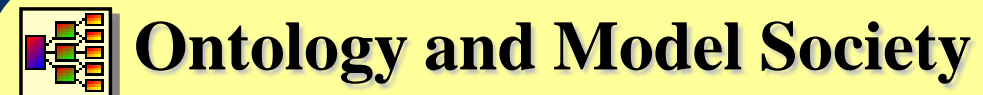

\section{Annotations Society}

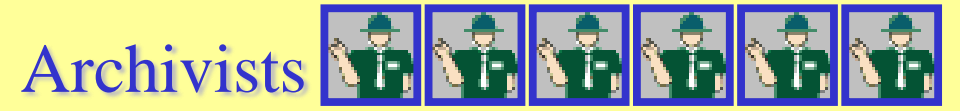

Ain

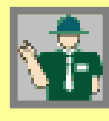

\section{Ontologist Agents}
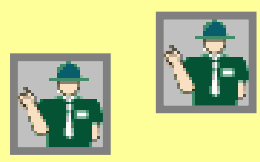

Federated

Matchmakers
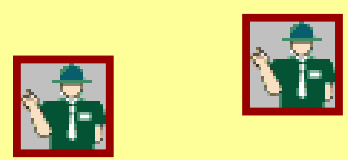

$4=$

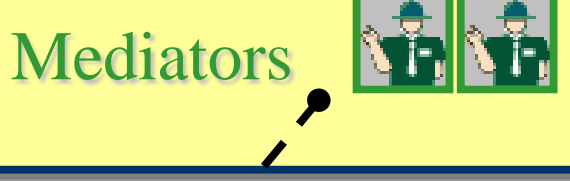

Interconnection Society

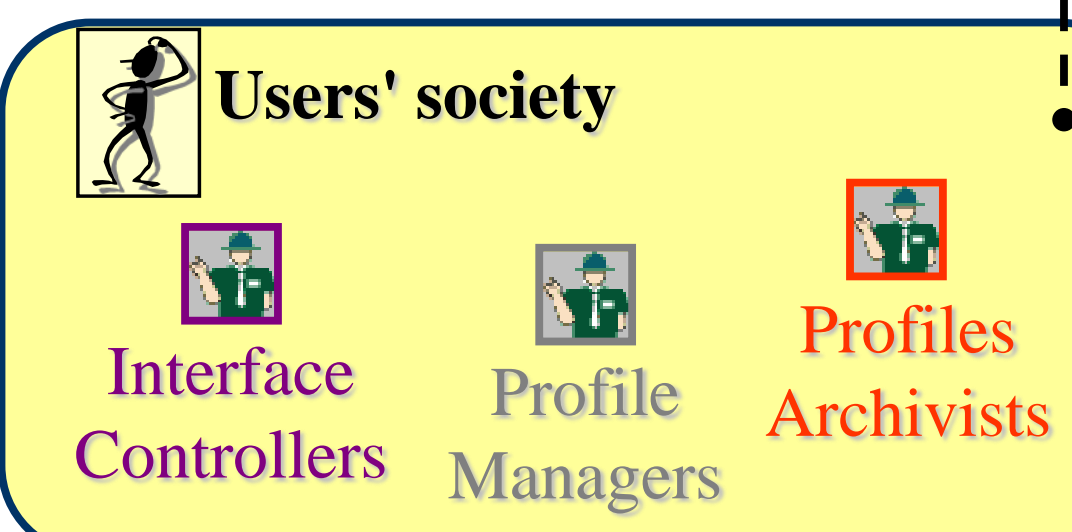




\section{- Societies of replication and serving}

- Ontologist: to store and provide ontology

\begin{tabular}{|l|l|}
\hline role model & $\begin{array}{l}\text { Ontology Archivist role in the Ontology and } \\
\text { Model society }\end{array}$ \\
\hline responsibilities & store and retrieve O'CoMMA \\
\hline collaborators & Directory facilitator, Interface Controller, \\
& User Profile Manager, User Profile Archivist, \\
& Annotation Mediator, Annotation Archivist, \\
\hline external interfaces & RDFS schemas manipulation interface \\
\hline relationships & - \\
\hline expertise & ontology management an querying \\
\hline interactions & Query-Ref, Request and Subscribe; FIPA ACI \\
\hline others & -
\end{tabular}

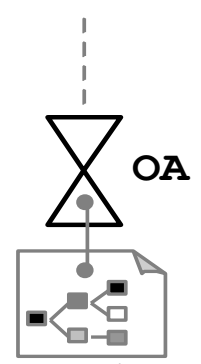

- Corporate model archivist: to store and provide structural model of the (human) organisation

\begin{tabular}{|l|l|}
\hline role model & $\begin{array}{l}\text { Corporate Model Archivist role in the Ontology } \\
\text { and Model society }\end{array}$ \\
\hline responsibilities & store, query, retrieve the organisational model \\
\hline collaborators & $\begin{array}{l}\text { Directory facilitator, Interface Controller, } \\
\text { Annotation Mediator, Ontology Archivist }\end{array}$ \\
\hline external interfaces & RDF annotatlon manlpulatlon lntertace \\
\hline relationships & composition: management and annotation archivist \\
\hline expertise & annotation management an querying \\
\hline interactions & Query-Ref, Request, Subscribe; FIPA ACL \\
\hline others & -
\end{tabular}

directory facilitator

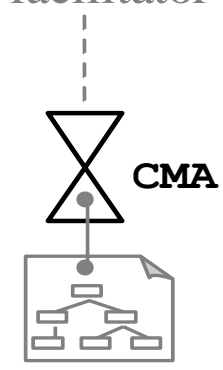

corporate model 


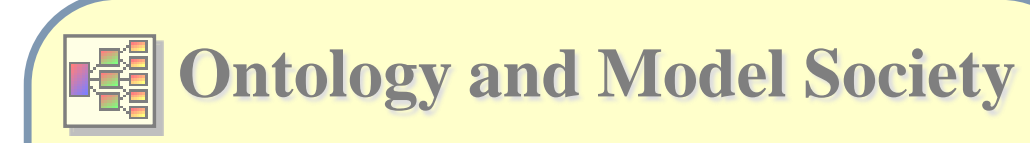

$\exists$ Annotations Society

in.

Ontologist Agents

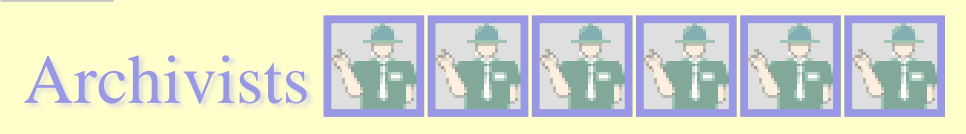

(it)
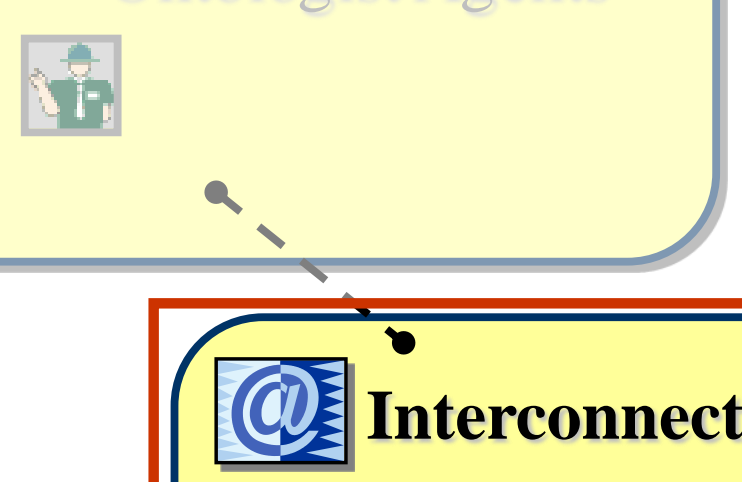

Interconnection Society
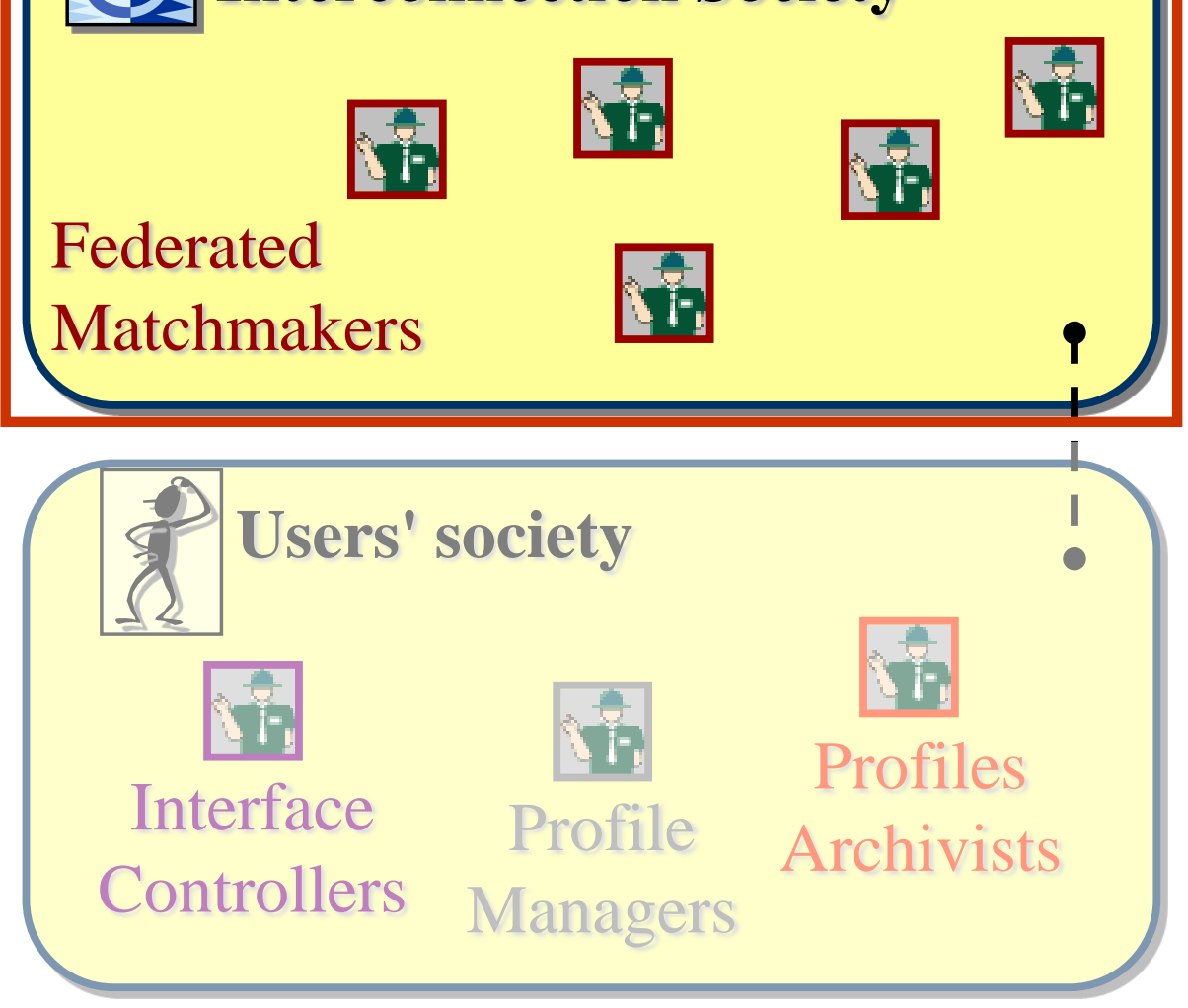

Fabien.Gandon@sophia.inria.fr 


\section{- The Connection-dedicated sub-society:}

- Yellow pages service management

- Federable to allow us to configure the MAS

- Cooperating matchmakers (DF)

\begin{tabular}{|c|c|c|}
\hline role model & $\begin{array}{l}\text { Directory Facilitator role in the Yellow and } \\
\text { White pages society }\end{array}$ & \\
\hline responsibilities & $\begin{array}{l}\text { handles matchmaking among agents providing } \\
\text { registering and de-registering services as well } \\
\text { as service querying }\end{array}$ & $*$ \\
\hline collaborators & $\begin{array}{l}\text { Ontology Archivist, Interface Controller, User } \\
\text { Profile Manager, User Profile Archivist, } \\
\text { Annotation Mediator, Annotation Archivist, } \\
\text { Corporate Model Archivist }\end{array}$ & \\
\hline external interfaces & - & \\
\hline relationships & - & \\
\hline expertise & FIPA Service description management & ell \\
\hline interactions & $\begin{array}{l}\text { Request; FIPA ACL, FIPA-Agent-Management } \\
\text { ontology }\end{array}$ & \\
\hline others & (Provided by JADE) & \\
\hline
\end{tabular}




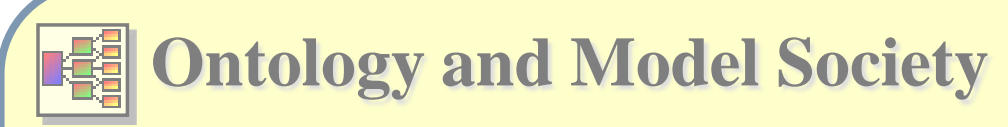

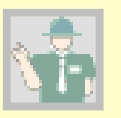

.

\section{Ontologist Agents}
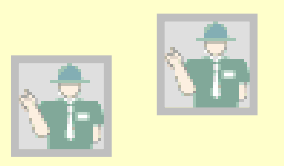

Federated

Matchmakers
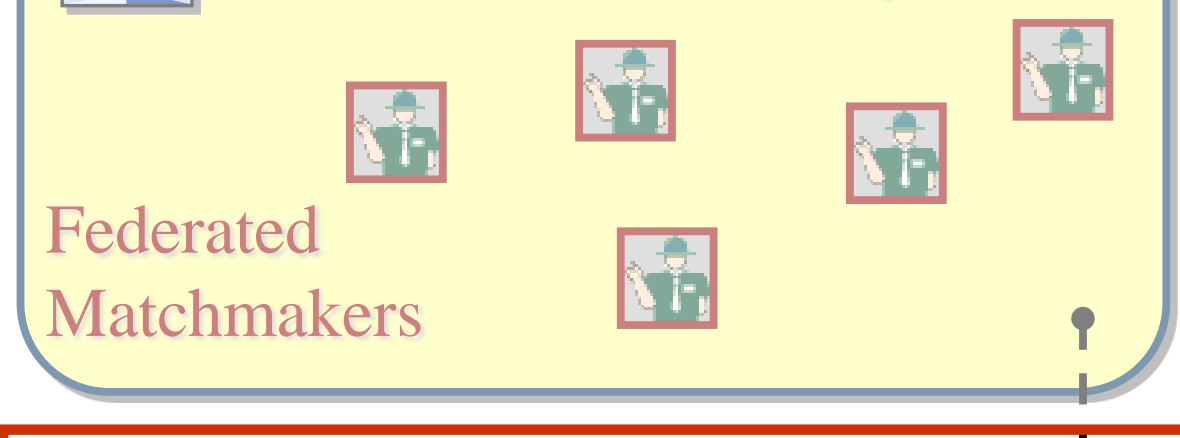

Annotations Society

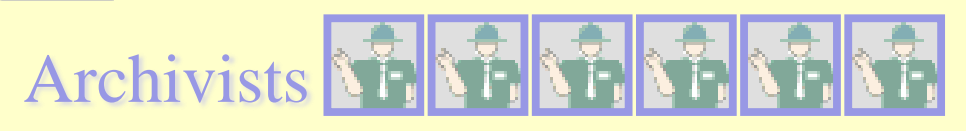

Interconnection Society

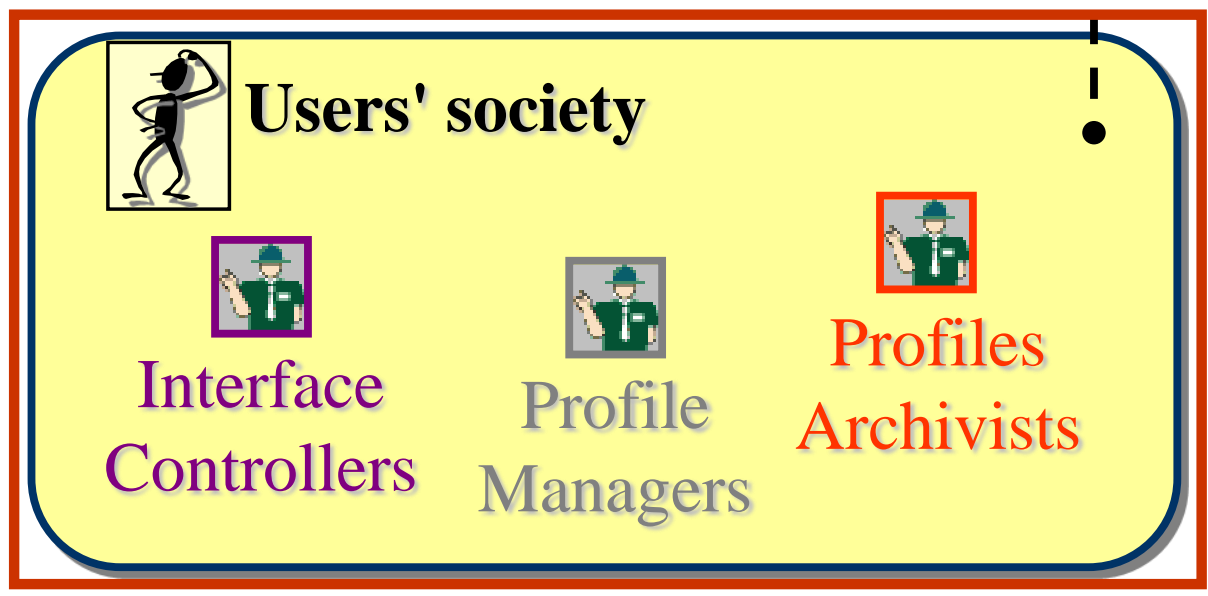

Fabien.Gandon@sophia.inria.fr 


\section{- Three main roles in user-dedicated sub-society:}

- GUI management: direct interactions with user (IC)

- User Profile management:

- Symbolic learning techniques (UPM)

- Archiving and ensuring secured login (UPA)

\begin{tabular}{|l|l|}
\hline role model & $\begin{array}{l}\text { Interface Controller role in the user-dedicated } \\
\text { society } \\
\text { handles interface between the user and the rest } \\
\text { of the multi-agent system }\end{array}$ \\
\hline responsibilities & $\begin{array}{l}\text { Ontology Archivist, User Profile Manager, } \\
\text { Corporate Model Archivist, Directory Facilitator }\end{array}$ \\
\hline collaborators & $\begin{array}{l}\text { graphic interfaces, HTML browser and XLM/XSLT } \\
\text { engines }\end{array}$ \\
\hline external interfaces \\
\hline relationships & - \\
\hline expertise & User interactions management \\
\hline interactions & Request; FIPA ACL \\
\hline others & not persistently running \\
\hline
\end{tabular}

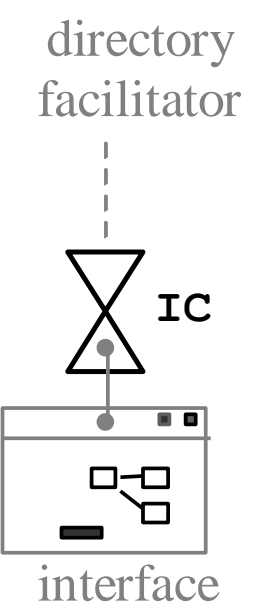




\section{- User profile manager: machine learning}

\begin{tabular}{|l|l|}
\hline role model & $\begin{array}{l}\text { User Profile Manager role in the user-dedicated } \\
\text { society }\end{array}$ \\
\hline responsibilities & $\begin{array}{l}\text { handles customisation and adaptation in the user } \\
\text { profile }\end{array}$ \\
\hline collaborators & $\begin{array}{l}\text { Ontology Archivist, Interface Controller, } \\
\text { Annotation Mediator, User Profile Archivist, } \\
\text { Directory Facilitator }\end{array}$ \\
\hline external interfaces & machine learning techniques libraries \\
\hline relationships & (partly absorbed the User profile processor) \\
\hline expertise & learning from usage patterns \\
\hline interactions & Request; FIPA ACL \\
\hline others & - \\
\hline
\end{tabular}

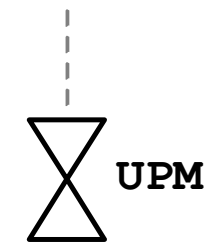

\section{- User profile archivist: profile storage and login}

\begin{tabular}{|l|l|}
\hline role model & $\begin{array}{l}\text { User Profile Archivist role in the user- } \\
\text { dedicated society }\end{array}$ \\
\hline responsibilities & handles storage and access in the user profile \\
\hline collaborators & $\begin{array}{l}\text { Ontology Archivist, Annotation Mediator, User } \\
\text { Profile Manager, Directory Facilitator }\end{array}$ \\
\hline external interfaces & RDF annotation manipulation interface \\
\hline relationships & composition: management and annotation archivist \\
\hline expertise & storage, access right and retrieval of profiles \\
\hline interactions & Request; FIPA ACL \\
\hline others & - \\
\hline
\end{tabular}

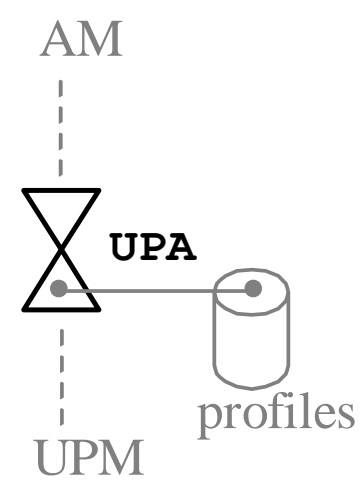




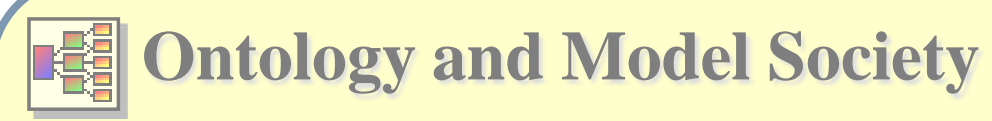

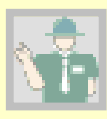

(3)

\section{Ontologist Agents}

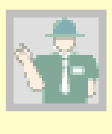

\section{$\bigoplus$ Annotations Society}

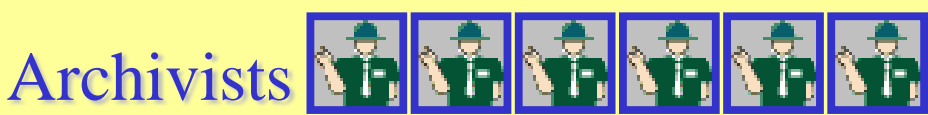

Mediators,
Federated

Matchmakers
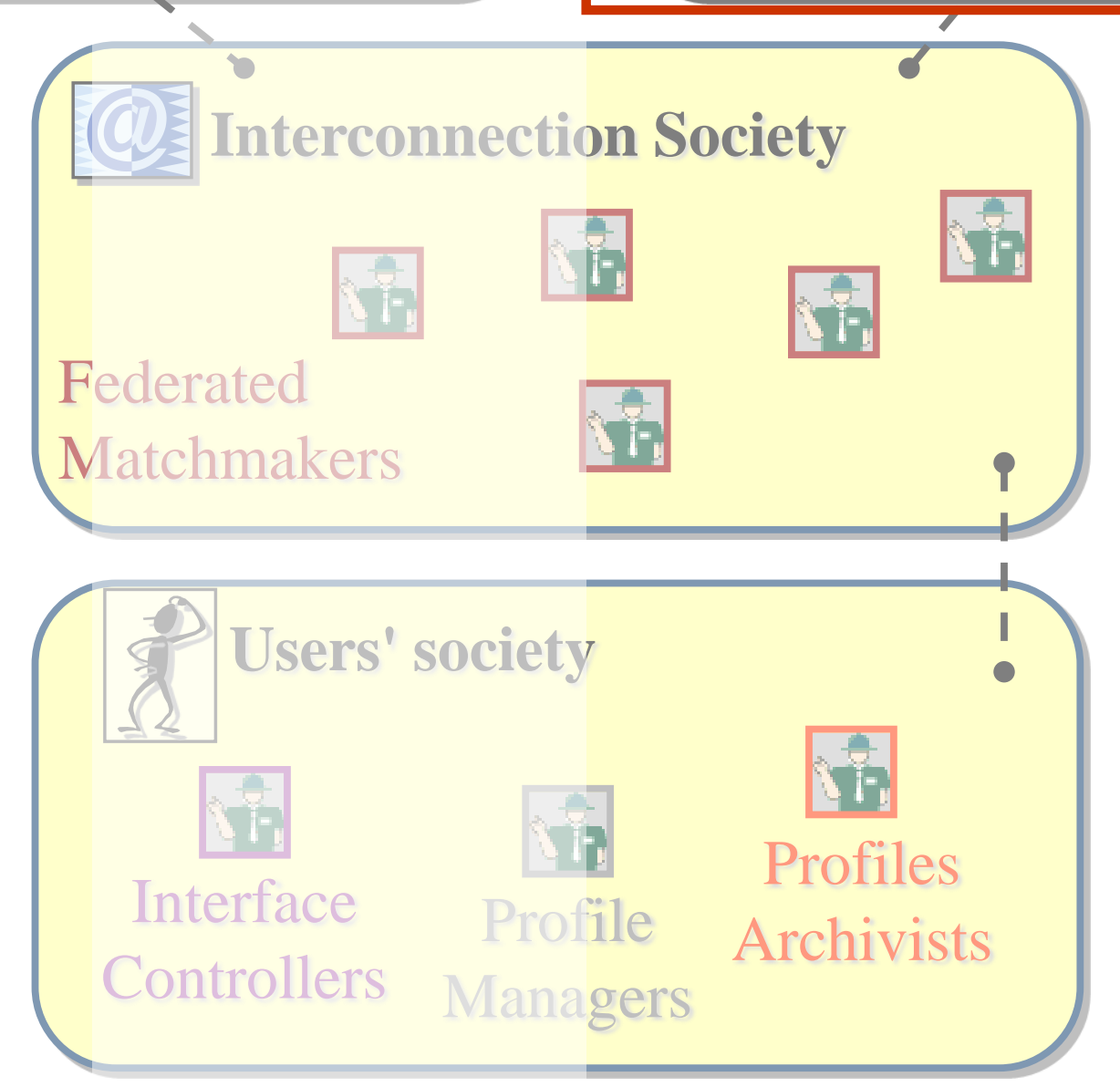

8 Interconnection Society 
- The annotation-dedicated sub-society:

- Distributed local sources

- Archive annotations on documents of the O.M.

- Search \& retrieve references matching queries

- Hierarchy: to federate two aspects of the society

- Mediator role (manage social aspect):

- supervising distributed query solving process

- managing allocation of new annotations

- notification of new annotations to trigger push functions

\begin{tabular}{|l|l|}
\hline role model & $\begin{array}{l}\text { Annotation Mediator role in the Annotation- } \\
\text { dedicated society }\end{array}$ \\
\hline responsibilities & $\begin{array}{l}\text { handle distribution of annotations over the } \\
\text { archivists both for new annotation submissions } \\
\text { and query solving processes }\end{array}$ \\
\hline collaborators & $\begin{array}{l}\text { Directory facilitator, User Profile Manager, } \\
\text { Ontology Archivist, Annotation Archivist, } \\
\text { Corporate Model Archivist }\end{array}$ \\
\hline external interfaces & RDF annotation manipulation interface \\
\hline relationships & - \\
\hline expertise & query and submission management \\
\hline interactions & $\begin{array}{l}\text { Query-Ref, Contract-Net, Subscribe, Request; } \\
\text { FIPA ACL }\end{array}$ \\
\hline others & - \\
\hline
\end{tabular}

directory

facilitator

Fabien.Gandon@sophia.inria.fr

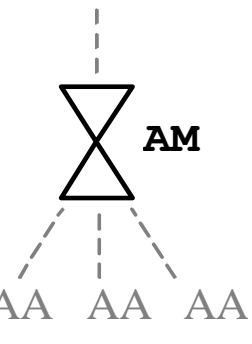


- The annotation-dedicated sub-society:

- Distributed local sources

- Archive annotations on documents of the O.M.

- Search \& retrieve references matching queries

- Hierarchy: to federate two aspects of the society

- Archivist role (manage local aspect):

- attached to \& exploits local base

- answers to query \& proposes archiving services

\begin{tabular}{|l|l|}
\hline role model & $\begin{array}{l}\text { Annotation Archivist role in the Annotation- } \\
\text { dedicated society }\end{array}$ \\
\hline responsibilities & store and query the annotations of the memory \\
\hline collaborators & $\begin{array}{l}\text { Directory facilitator, Annotation Mediator, } \\
\text { Ontology Archivist }\end{array}$ \\
\hline external interfaces & RDF annotation manipulation interface \\
\hline relationships & $\begin{array}{l}\text { also part of the roles in Corporate Model } \\
\text { Archivist and User Profile Archivist }\end{array}$ \\
\hline expertise & annotation archiving an querying \\
\hline interactions & Query-Ref, Contract-Net; FIPA ACL \\
\hline others & - \\
\hline
\end{tabular}

annotation

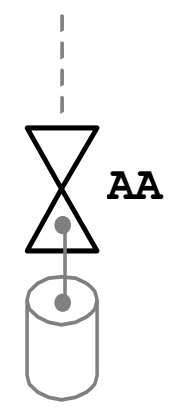

annotation archive 


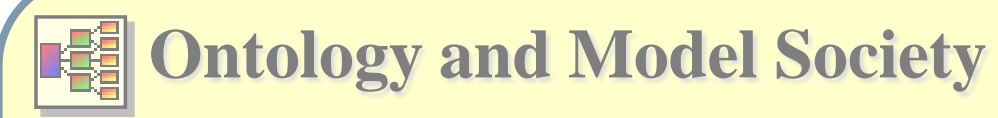

Annotations Society

in

Ontologist Agents

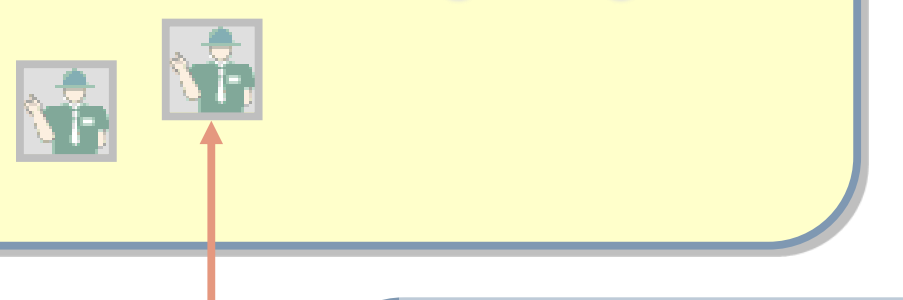

Interconnection Society

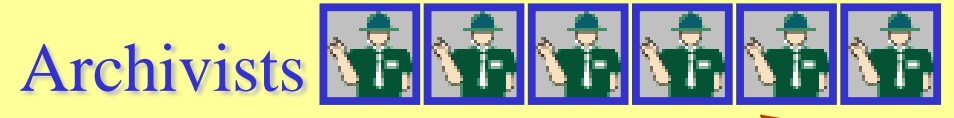

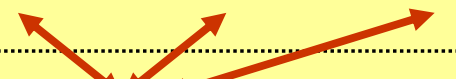

Mediators

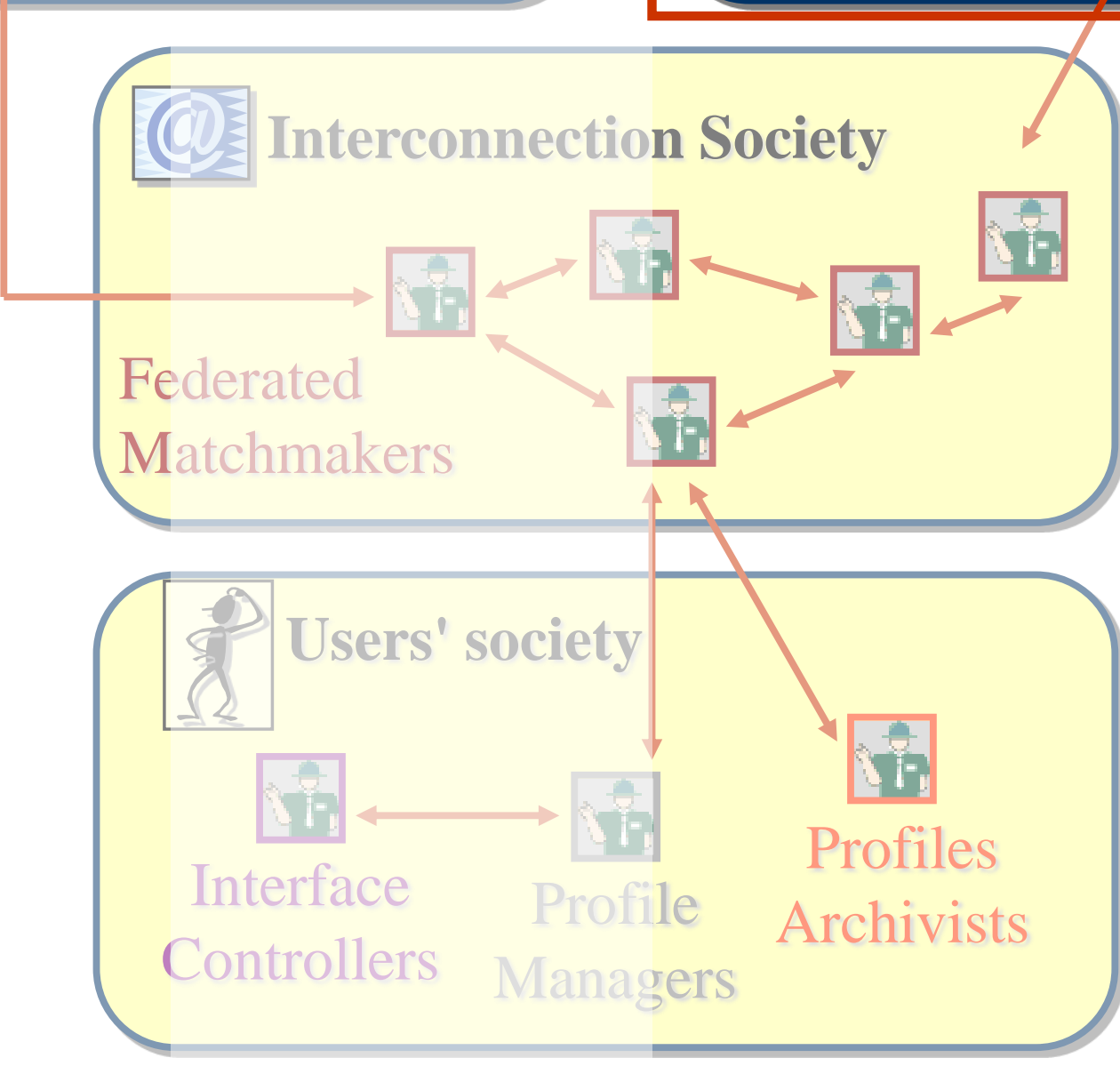

Fabien.Gandon@sophia.inria.fr 
C-Net : Annotation allocation

- Mediator \& Archivists discuss best place to archive

- Contract-net (CfP, Proposal, Accept/Reject):

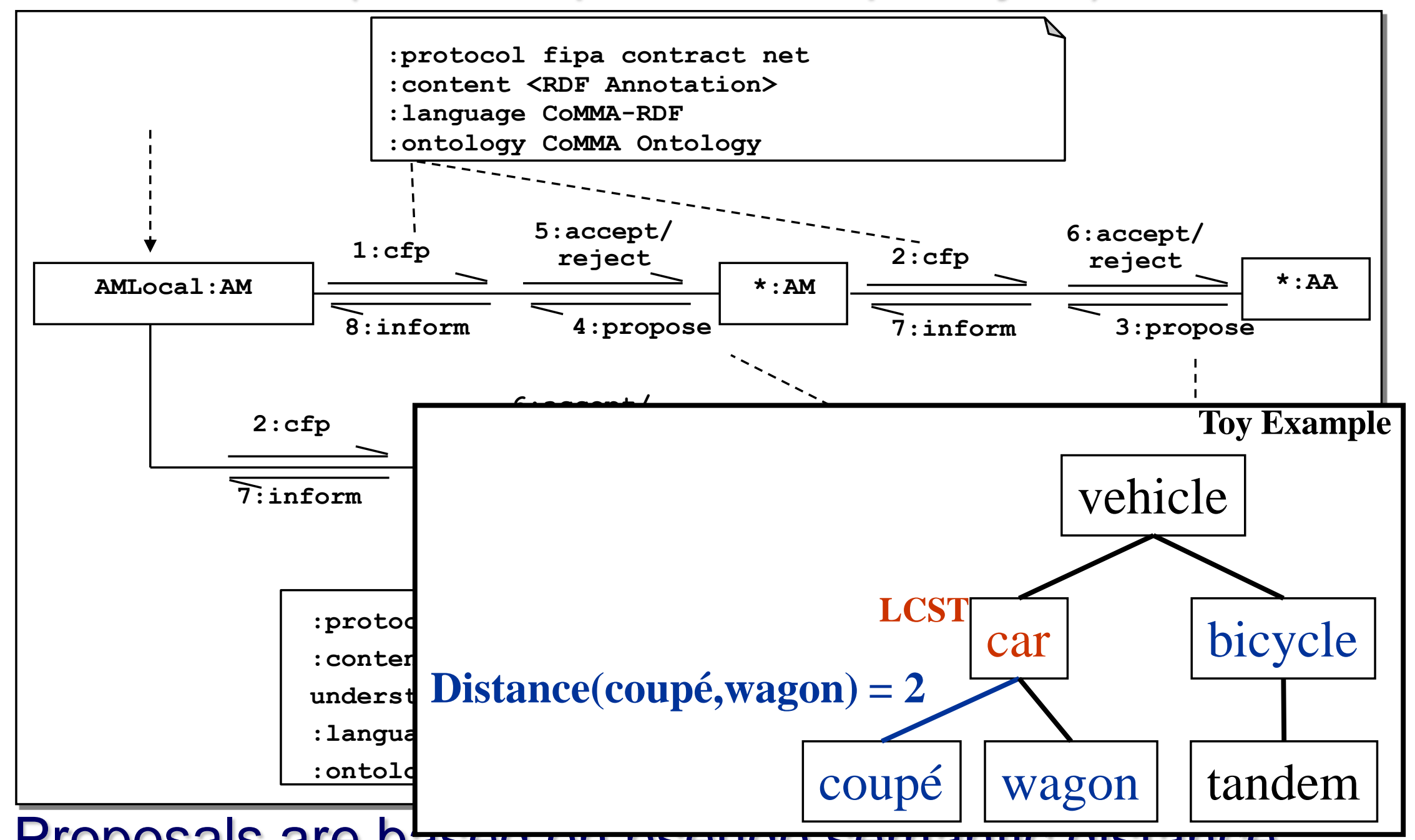

- Proposals are baseu uाrpseuuo semriantic uistailce over ontology space 


\section{- Fragmentation et distributed queries}

- Co-operatively solve a query (multi-stage Query-Ref):

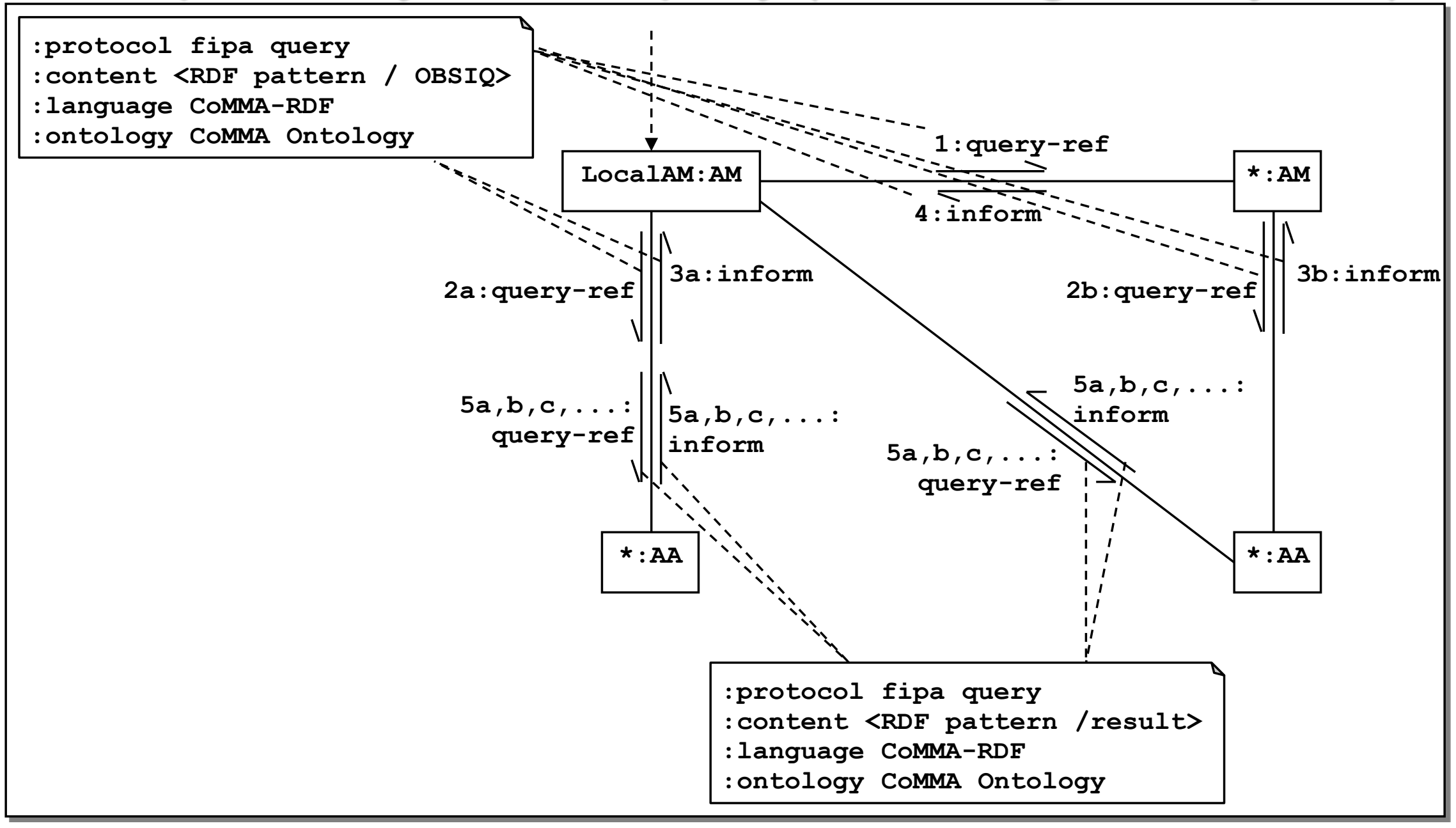

- Query decomposition using URIs as cut/join points

- Allocation of sub-query based on overlap description

- Ontology primitives usage statistics 


\section{(QUERY-REF}

sender ( agent-identifier

:name localUPM@fapollo:1099/JADE)

:receiver (set ( agent-identifier

:name AM@fapollo:1099/JADE) )

:content

((all ?x (is-answer-for

(query

:pattern "<?xml version =|"1.0|"?> <rdf:RDF xml:lang="en" xmlns:rdf= "http://www.w3.org/1999/02/22-rdf-syntax-ns\#"

xmlns:comma="http://www.inria.fr/acacia/comma\#">

<comma:Memo><comma:Designation>?</comma:Designation>

$<$ comma:Memo $>$

$<$ (rdf:RDF>") ? ) ) )

:reply-with QuerylocalUPM987683105872

:language CoMMA-RDF

:ontology CoMMA-annotation-ontology

:protocol FIPA-Query

:conversation-id QuerylocalUPM987683105872 


\section{(QUERY-REF}

:sender ( agent-identifier

:name localUPM@fapollo:1099/JADE)

:receiver (set ( agent-identifier

:name AM@fapollo:1099/JADE) )

:content

((all ?x (is-answer-for

(query

:pattern "<?xml version =|"1.0|"?> <rdf:RDF xml:lang="en" xmlns:rdf= "http://www.w3.org/1999/02/22-rdf-syntax-ns\#"

xmlns:comma="http://www.inria.fr/acacia/comma\#">

<comma:Memo><comma:Designation>?</comma:Designation>

$<$ comma:Memo>

$<$ (rdf:RDF>") ? x ) ) )

:reply-with QuerylocalUPM987683105872

:language CoMMA-RDF

:ontology CoMMA-annotation-ontology

:protocol FIPA-Query

:conversation-id QuerylocalUPM987683105872 


\section{(QUERY-REF}

sender ( agent-identifier

:name localUPM@fapollo:1099/JADE)

:receiver (set ( agent-identifier

:name AM@fapollo:1099/JADE) )

:content

\section{((all ?x (is-answer-for}

\section{(query}

:pattern "<?xml version =|"1.0|"?> <rdf:RDF xml:lang="en" xmlns:rdf= "http://www.w3.org/1999/02/22-rdf-syntax-ns\#" xmlns:comma="http://www.inria.fr/acacia/comma\#"> <comma:Memo><comma:Designation>?</comma:Designation> $</$ comma:Memo $>$ $<$ (rdf:RDF>") ?x ) ) )

:reply-with QuerylocalUPM987683105872

:language CoMMA-RDF :ontology CoMMA-annotation-ontology :protocol FIPA-Query :conversation-id QuerylocalUPM987683105872 


\section{(QUERY-REF}

sender ( agent-identifier

:name localUPM@fapollo:1099/JADE)

:receiver (set ( agent-identifier

:name AM@fapollo:1099/JADE) )

:content

((all ?x (is-answer-for

(query

:pattern "<? xml version =|"1.0|"?> <rdf:RDF xml:lang="en" $x$ mlns:rdf= "http://www.w3.org/1999/02/22-rdf-syntax-ns\#" xmlns:comma="http://www.inria.fr/acacia/comma\#">

<comma:Memo><comma:Designation>?</comma:Designation>

$</$ comma:Memo $>$

$<$ (rdf:RDF>") ?x ) ) )

:reply-with QuerylocalUPM987683105872

:language CoMMA-RDF

:ontology CoMMA-annotation-ontology

:protocol FIPA-Query

:conversation-id QuerylocalUPM987683105872 


\section{- Ontology as a cornerstone of DAI for KM}

- Modelling and representation consensus

- Communication between humans (ontology as a component of the memory, annotations and models)

- Communication between agents (c.f. message)

- Formal structures (annotations, schemata, etc.) for inferences

- Ontological consensus to build consensus above:

- computational consensus, e.g.: measure

- co-operation consensus, e.g.: protocols

- Vice-versa: DAl as a cornerstone for ontologies

- Complementary domains

-D.A.I. $\leftrightarrow$ Ontology

- D.A.I. $\leftrightarrow$ Semantic Web

- Ontology $\leftrightarrow$ Semantic Web 
- Two trials and one open-day

- Results \& feedback:

: : Ergonomics problems

(2) Specialization problems

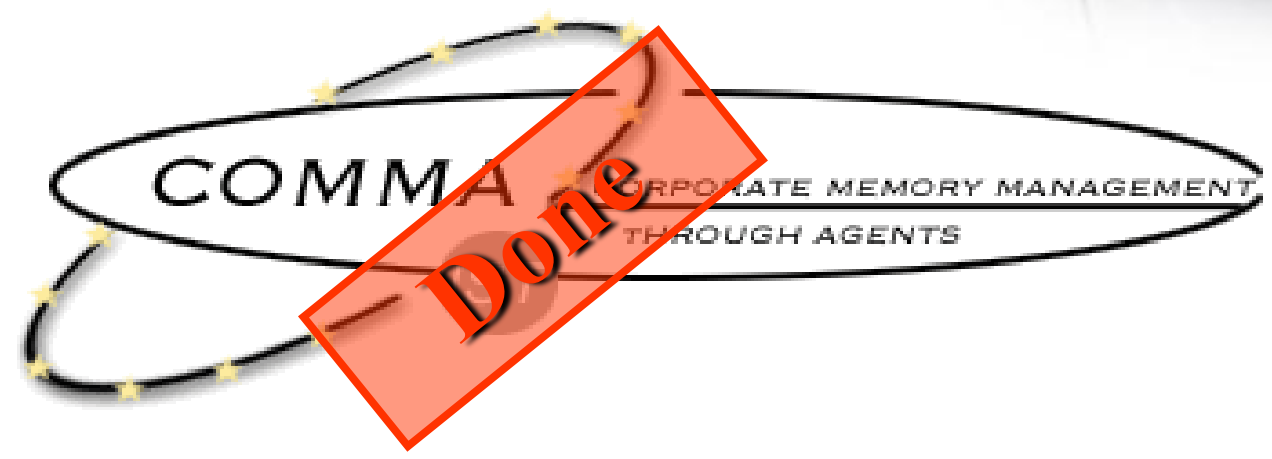

(2) Monolithic solution evaluation problem

(2) No large-scale evaluation (groups of $4 / 6$ users)

(:) Proof of concept: usability and usefulness recognized

(;) Integration of results from different domains

(2) Developers appreciation of ontology-agent approach

(-) Industrial interest in the development of the prototype

- On going improvements in the team:

- pseudo distance \& decomposition algorithms

- Web mining wrappers: semi-automatic annotation

- Ontology mapping: connect 2 corporate semantic webs

- Ontology reuse and integration and NLP tools 


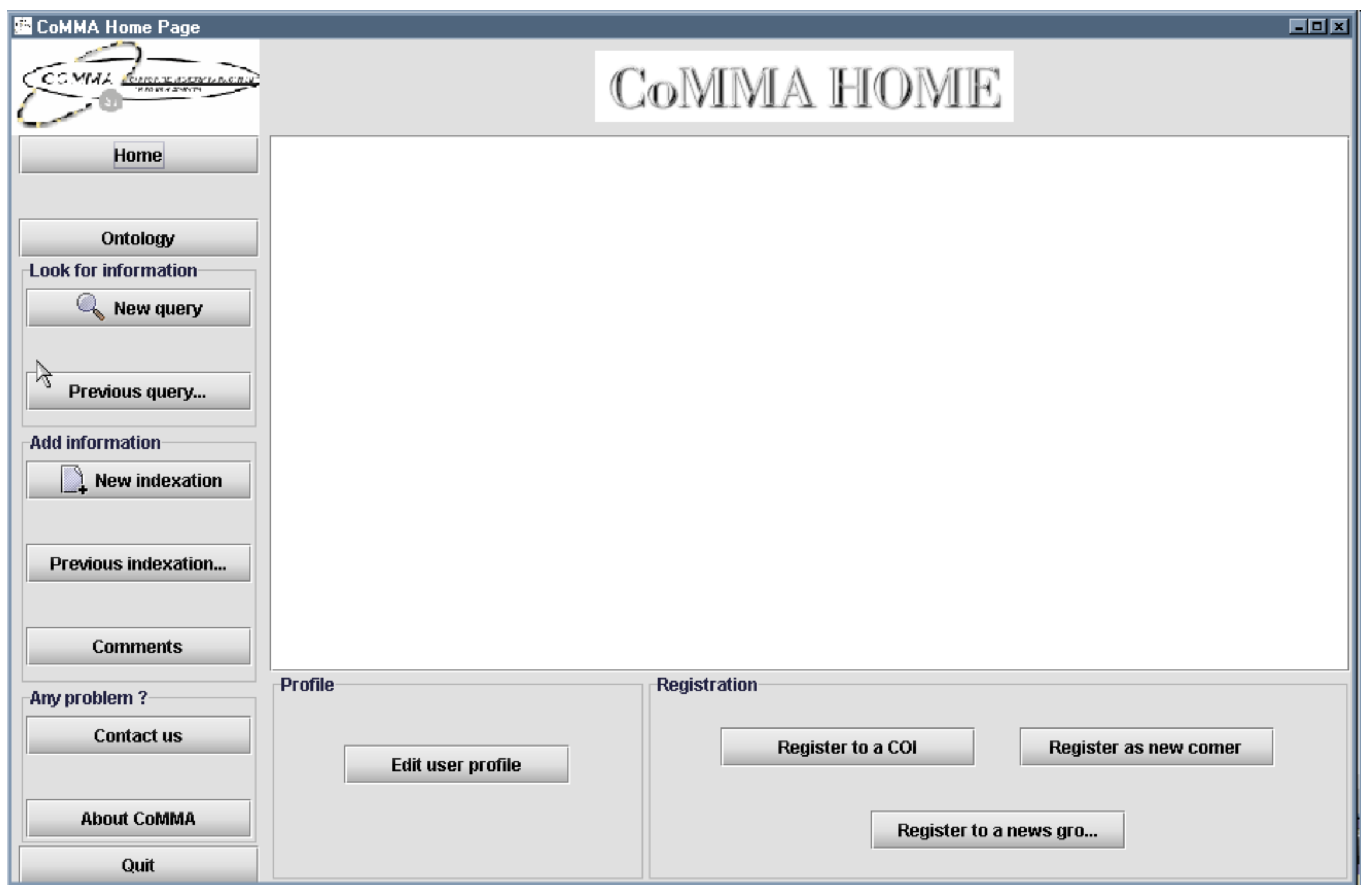

Fabien.Gandon@sophia.inria.fr 


\section{Annotation allocation: contract-net based on pseudo-semantic distance bids}

3s sniffer0effapollo:1099/JADE - Sniffer Agent

$-\mid$ a $x$

Actions About

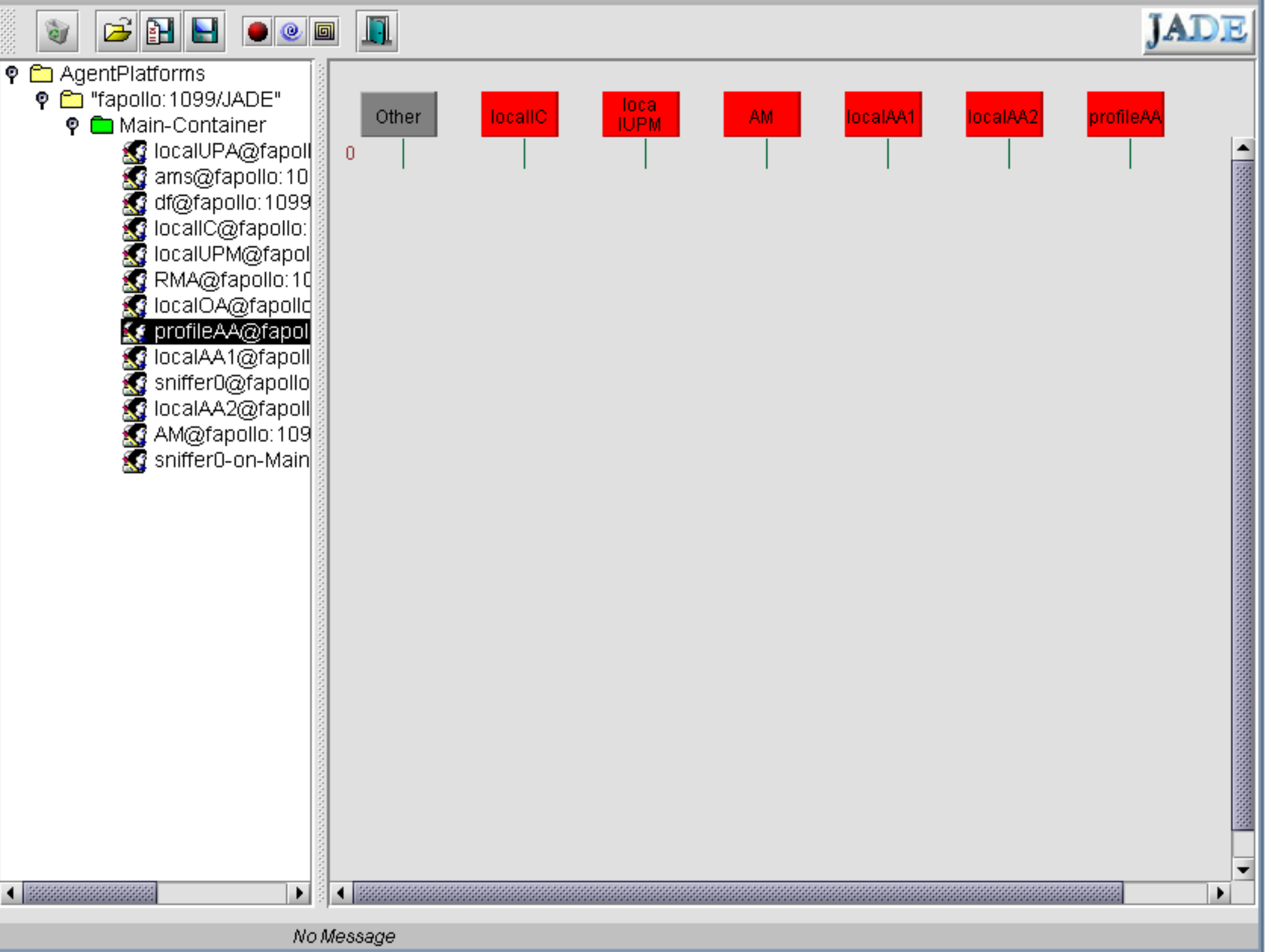

Fabien.Gandon@sophia.inria.fr 


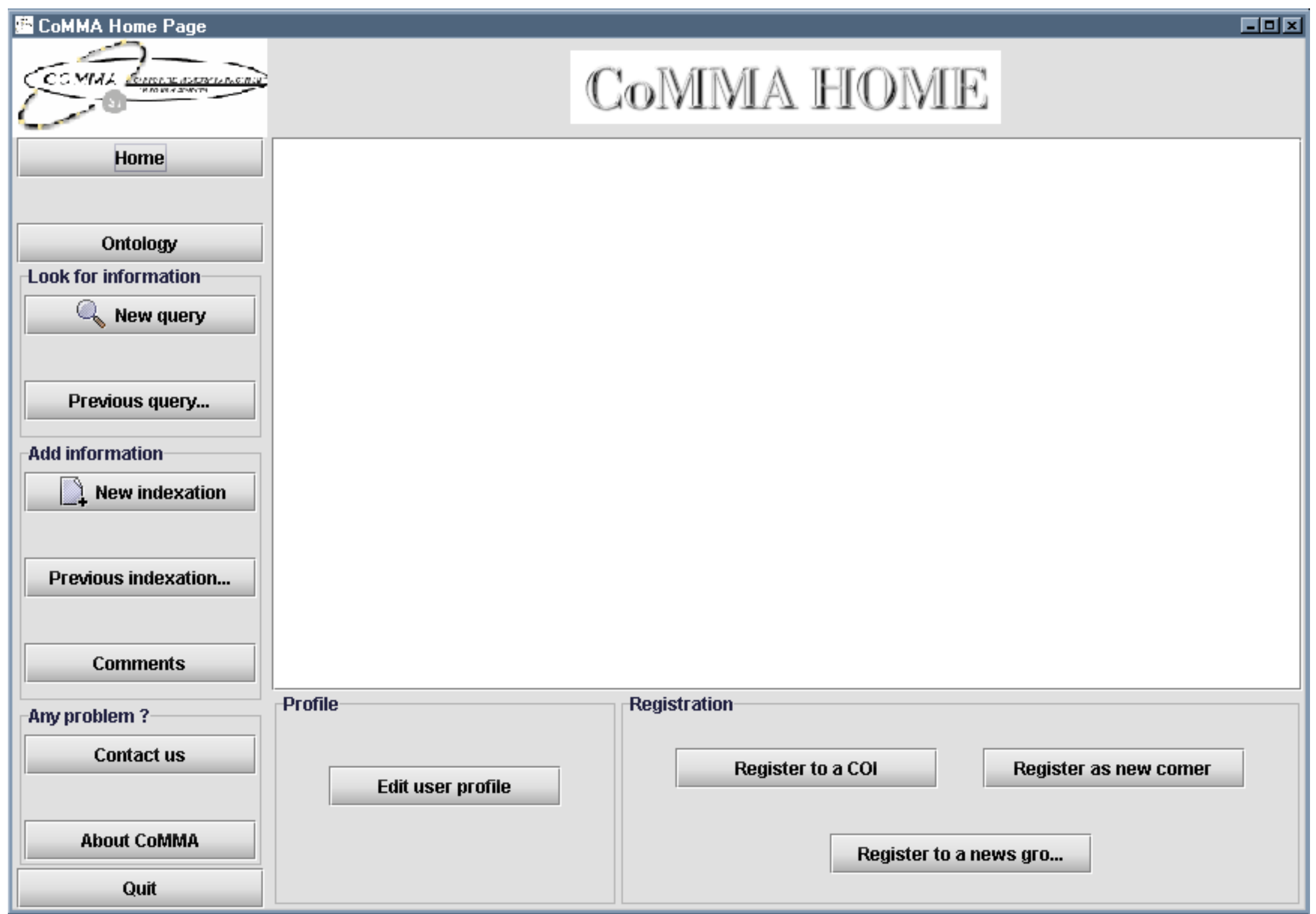

Fabien.Gandon@sophia.inria.fr 
S5 sniffer0@(fapollo:1099/JADE - Sniffer Agent

Actions About

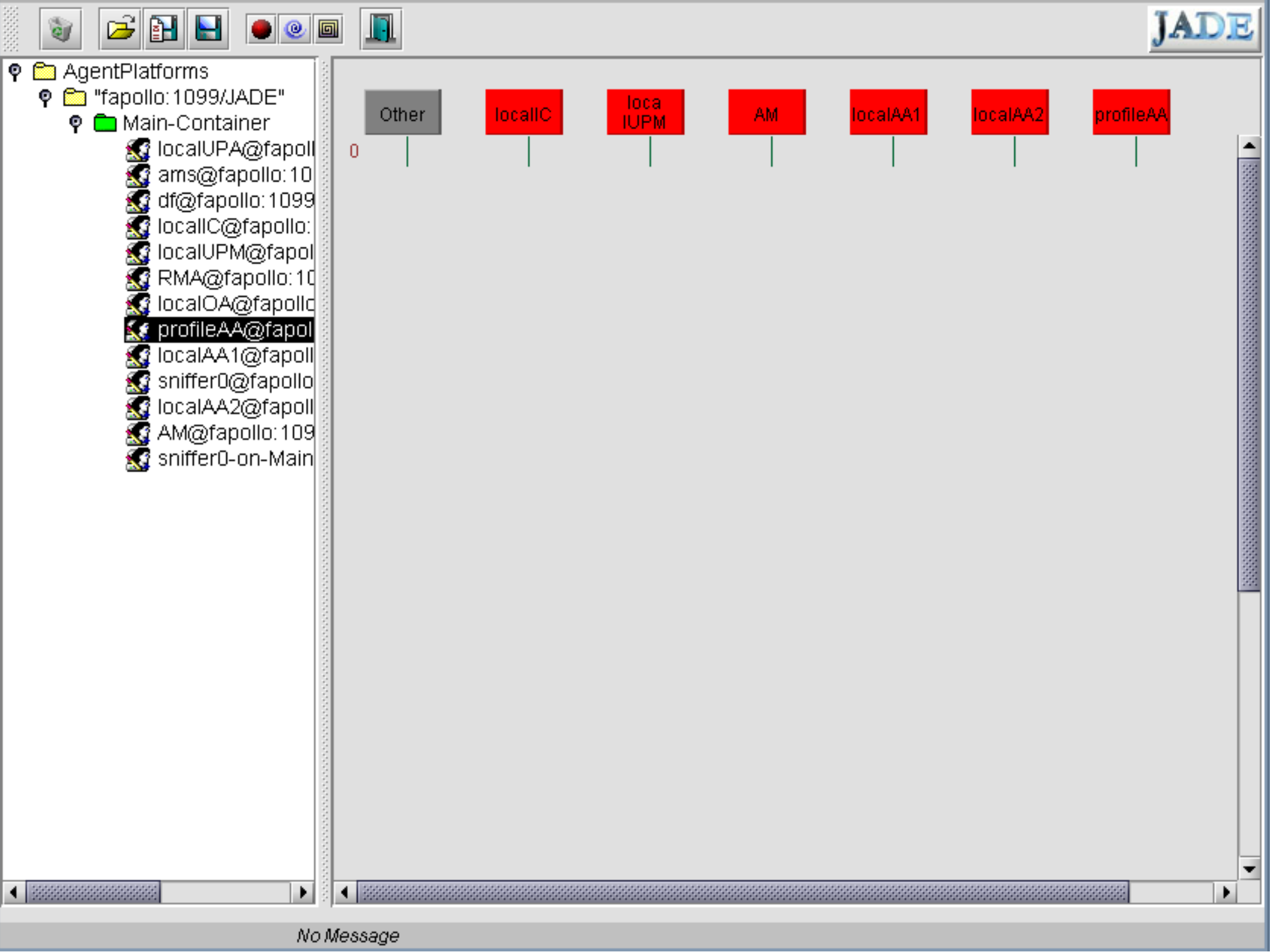

Fabien.Gandon@sophia.inria.fr 
(C) transparency show http://www-sop.inria. fr/acacia/personnel/Fabien.Gandon/cstb.ppt (?)

모 target:

(C) organizational entity

国 is interested by:

(c) knowledge management (iv)

[- title: Presentation of CoMMA to the CSTB

(C) transparency show

http://www-sop.inria. fr/acacia/personnel/Fabien. Gandon/research/ekaw200 fhinkaw.ppt (?)

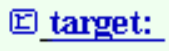

(C) organizational entity

⿴囗玉 is interested by:

(C) knowledge management (iv)

[모 title: Presentation of CoMMA Methodology at EKAW2000

(C) presentation http://fapollo:8080/comma/doc/CoMMATerms.avi (?)

모롤

(C) organizational entity

[r] is interested hy. 


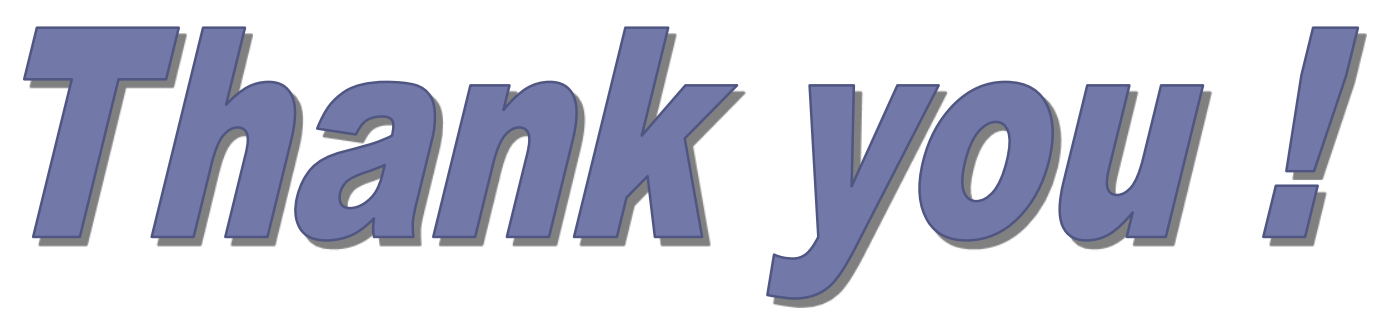

Fabien.Gandon@sophia.inria.fr 